Assurances et gestion des risques

Insurance and Risk Management

\title{
Amélioration de la méthodologie de construction de la courbe des taux sans risque dans la zone UEMOA
}

\section{Florent Gbongué}

Volume 86, numéro 2-3, décembre 2019

URI : https://id.erudit.org/iderudit/1068504ar

DOI : https://doi.org/10.7202/1068504ar

Aller au sommaire du numéro

\section{Éditeur(s)}

Faculté des sciences de l'administration, Université Laval

\section{ISSN}

1705-7299 (imprimé)

2371-4913 (numérique)

Découvrir la revue

\section{Citer cet article}

Gbongué, F. (2019). Amélioration de la méthodologie de construction de la courbe des taux sans risque dans la zone UEMOA. Assurances et gestion des risques / Insurance and Risk Management, 86(2-3), 127-164.

https://doi.org/10.7202/1068504ar
Résumé de l'article

Dans la zone de l'Union économique et monétaire ouest-africaine (UEMOA), la construction d'une courbe des taux sans risque est un objectif pour les décideurs publics. En effet, elle permet d'accompagner les mutations réglementaires actuelles, ainsi que le processus de développement du marché financier régional (MFR). En s'appuyant sur les conclusions de Gbongué et Planchet (2015) et Gbongué (2019), nous proposons une nouvelle méthodologie de construction de la courbe des taux sans risque, adaptée aux particularités de cette zone, dans l'optique de réduire les erreurs d'estimation de la valeur théorique des obligations souveraines. Notons qu'elle s'appuie sur les fondamentaux du modèle de Nelson et Siegel, dans le but de faciliter la prévision de cette courbe dans le futur.
Tous droits réservés $\odot$ Faculté des sciences de l'administration, Université Laval, 2020
Ce document est protégé par la loi sur le droit d'auteur. L’utilisation des services d'Érudit (y compris la reproduction) est assujettie à sa politique d'utilisation que vous pouvez consulter en ligne.

https://apropos.erudit.org/fr/usagers/politique-dutilisation/ 


\title{
AMÉLIORATION DE LA MÉTHODOLOGIE DE CONSTRUCTION DE LA COURBE DES TAUX SANS RISQUE DANS LA ZONE UEMOA
}

\author{
Florent GBONGUÉ, PhD1 \\ Université Félix-Houphouët-Boigny (UFHB) \\ FINAIR Conseil
}

\section{RÉSUMÉ}

Dans la zone de l'Union économique et monétaire ouest-africaine (UEMOA)², la construction d'une courbe des taux sans risque est un objectif pour les décideurs publics. En effet, elle permet d'accompagner les mutations réglementaires actuelles, ainsi que le processus de développement du marché financier régional (MFR). En s'appuyant sur les conclusions de Gbongué et Planchet (2015) et Gbongué (2019), nous proposons une nouvelle méthodologie de construction de la courbe des taux sans risque, adaptée aux particularités de cette zone, dans l'optique de réduire les erreurs d'estimation de la valeur théorique des obligations souveraines. Notons qu'elle s'appuie sur les fondamentaux du modèle de Nelson et Siegel, dans le but de faciliter la prévision de cette courbe dans le futur.

JEL Codes : C0, C6, E4, G1, H6.

Mots-clefs : UEMOA, courbe des taux sans risque, mutations réglementaires, marché financier régional, erreurs d'estimation, fondamentaux, Nelson et Siegel, prévision.

\section{ABSTRACT}

In the West African Economic and Monetary Union (WAEMU) zone, the construction of a risk-free interest rate curve is an objective for public decision-makers. Indeed, it helps to support the current regulatory changes, as well as the development process of the regional financial market. Based on the conclusions of Gbongué and Planchet (2015) and Gbongué (2019), we propose a new methodology for constructing the risk-free interest rate curve, adapted to the particularities of this zone, with the aim of reducing estimation errors of the theoretical value of sovereign bonds. Note that it is based on the fundamentals of the Nelson and Siegel model, in order to facilitate the prediction of this curve in the future. 
Jel Codes: C0, C6, E4, G1, H6.

Keywords: UEMOA, risk-free interest rate curve, regulatory changes, regional financial market, estimation errors, fundamentals, Nelson and Siegel, prediction.

\section{INTRODUCTION}

Par définition, la courbe des taux sans risque ou de rendement (yield curve) est la fonction qui, à une date donnée, pour chaque maturité, indique le niveau de taux d'intérêt pour un placement sans risque de défaut de l'émetteur. Malgré les applications multiples ${ }^{3}$ de cette courbe, nous remarquons son inexistence dans plusieurs espaces économiques africains ( $c f$. AFMI $^{4}$ [2016]). Dans la zone UEMOA, l'absence d'une courbe des taux ${ }^{5}$ officielle a un impact négatif sur son développement. En effet, le marché des capitaux tarde à se développer, malgré l'augmentation des émissions souveraines ${ }^{6}$, en raison de l'existence des asymétries d'information. Cette situation limite la mobilisation des ressources sur le $\mathrm{MFR}^{7}$ et favorise une hausse du coût de financement. Au niveau de la réglementation, des mutations importantes ont été observées, notamment l'application du dispositif Bâle II et III pour les banques et le Système comptable OHADA (SYSCOHADA) révisé pour les entreprises. En pratique, elles visent à assurer la convergence vers les normes internationales, afin d'harmoniser les pratiques.

L'application de ces réformes requiert souvent de déterminer les facteurs d'actualisation, afin de déterminer la valeur actuelle d'une série future de flux financiers. Comme exemple, nous avons la valorisation des actifs financiers, qui modifie la qualité des informations financières de l'entreprise. De plus, ils pourront être utilisés par les assureurs de la zone de la Conférence interafricaine des marchés d'assurance (CIMA) ${ }^{8}$, pour estimer leurs engagements dans une logique best estimate, ce qui contribuera à proposer la politique d'investissement adéquate.

Dans le secteur bancaire, la courbe des taux est cruciale dans l'élaboration d'un système interne de tarification. Rappelons qu'elle contient également une information et son comportement peut renseigner sur l'évolution de l'économie ( $c f$. Stambaugh [1998], Svensson [1994b], Frankel et Lown [1994]). Pour les économistes, l'inversion de cette courbe peut présager une récession économique. Selon Svensson (1994b), les taux à terme peuvent être des indicateurs avancés pour les anticipations d'inflation, de change et la reprise économique, sous certaines hypothèses. Ils constituent donc une information importante 
pour les autorités monétaires, qui doivent décider de la politique à mener. Ainsi, la majorité des banques centrales suivent attentivement son évolution, car elle peut permettre de comprendre l'impact de leurs politiques.

Dans la littérature, il existe plusieurs travaux scientifiques portant sur la construction de la courbe des taux dans la zone UEMOA. Pour preuve, nous pouvons citer Gbongué et Planchet (2015), Gbongué et autres (2017) et Gbongué (2019). Ces auteurs se sont intéressés à la comparaison des modèles de Nelson Siegel (1987), Svensson (1994) et Björk et Christensen (1999). La synthèse de ces travaux révèle que l'ajustement des données de la zone UEMOA requiert un modèle contenant un nombre important de paramètres, comme les modèles de Svensson et Björk et Christensen. Toutefois, Gbongué (2019) recommande l'utilisation du modèle de Nelson et Siegel dans une perspective de prévision, dans l'optique d'avoir des courbes de taux stables dans le futur.

De ce qui précède, nous proposons une autre méthodologie de construction de la courbe des taux sans risque de la zone UEMOA, dans le but d'améliorer la qualité d'ajustement des données existantes, par comparaison aux modèles candidats retenus dans Gbongué (2019). Ce modèle s'appuie sur les fondamentaux du modèle de Nelson et Siegel, car il contient également trois principaux facteurs, au lieu de quatre9 observés dans les modèles de Svensson et Björk et Christensen. En ne remettant pas en cause la qualité des autres modèles, les résultats révèlent que ce modèle semble adapter au contexte de l'UEMOA, en ce sens qu'il permet de capter les distorsions de court terme et réduire les erreurs d'estimation de la valeur théorique des obligations souveraines, de l'ordre de $10 \%, 2 \%$ et $9 \%$ en moyenne, par rapport aux modèles de Nelson et Siegel, Svensson et Björk et Christensen. De plus, le choix de ce modèle facilite la prévision de cette courbe dans l'avenir, répondant ainsi aux enjeux de la réglementation et du développement du marché des capitaux ${ }^{10}$.

Notre étude est organisée de la façon suivante:

- La section 2 présente la littérature sur les modèles de construction de la courbe des taux sans risque.

- La section 3 s'intéresse aux aspects méthodologiques.

- La section 4 porte sur l'analyse des résultats empiriques obtenus. 
- La section 5 est consacrée à la conclusion et aux perspectives de recherche futures.

- Les sections 6 et 7 contiennent respectivement la bibliographie et les annexes.

\section{REVUE DE LITTÉRATURE}

En règle générale, la détermination de la courbe des taux sans risque requiert de modéliser la valeur théorique du prix des obligations souveraines. Pour ce faire, plusieurs modèles de taux d'intérêt existent dans la littérature. Toutefois, les banques centrales ${ }^{11}$ privilégient surtout les modèles de type splines et paramétriques pour ajuster le facteur d'actualisation. La première catégorie comprend la spline quadratique (cf. McCulloch [1971], McCulloch et Kochin [1998]), la spline cubique ( $c f$. Mc Culloch [1975], Fisher et al. [1995]), la spline exponentielle ( $c f$. Vasicek et Fong [1982]), la B-splines (cf. Shea [1985], Steeley [1991]), sans oublier les travaux de Waggoner (1997), Deacon et Derry (1994), Bliss (1997), Anderson et Sleath (2001), etc. Ces méthodes ont été critiquées, car elles possèdent des propriétés économiques indésirables et sont souvent perçues comme des «boites noires" ( $c f$. Seber et Wild [2003]). Notons que Carriere (1998) souligne que les modèles de survie utilisés par les actuaires pour modéliser les pertes peuvent être considérés comme des modèles de prix. Dans ce cas, la fonction d'actualisation sera approximée par une fonction de survie.

En outre, la seconde catégorie contient les modèles paramétriques de type Nelson Siegel ( $c f$. Nelson et Siegel [1987], Svensson [1994]). Parmi ces modèles, le plus célèbre est le modèle de Nelson Siegel (1987). Depuis son apparition, il a été adopté par de nombreux experts du monde académique et professionnel. Il est aussi utilisé par les gestionnaires de portefeuille à revenu fixe ( $c f$. Barrett et autres [1995], Hodges et Parekh [2006], Martellini et Meyfredi [2007]). Dullmann et autres (2000) utilisent le modèle de Nelson et Siegel pour construire une courbe des taux zéro-coupon adaptée au marché allemand. Par contre, Fabozzi et autres (2005), Diebold et Li (2006) comparent plutôt les prévisions du modèle de Nelson et Siegel (NS) avec d'autres modèles. Ils trouvent que le modèle NS est plus précis sur le long terme. Enfin, Coroneo et autres (2008) utilisent plutôt les estimations des paramètres du modèle NS comme des paramètres d'un modèle affine (en l'absence d'opportunité d'arbitrage), pour construire la structure par terme des taux d'intérêt. 
Dans le contexte africain, il existe peu de travaux scientifiques qui portent sur la construction de la courbe des taux sans risque. Toutefois, nous pouvons citer Gbongué et Planchet (2015), Gbongué et autres (2017), Gbongué (2019), Muthoni et autres (2015), Lartey et Li (2018). En effet, Gbongué et Planchet (2015) proposent une méthodologie de construction d'une courbe des taux zéro-coupon, qui intègre le taux directeur, le taux de croissance réel de l'économie sur le long terme, ainsi que l'inflation cible (2\%). En comparant le modèle de Nelson et Siegel avec ses versions élargies, ils montrent que le modèle de Björk et Christensen s'ajuste le mieux aux données de la zone UEMOA à la date de cotation du 27/02/2015, même si les écarts des erreurs d'estimation par rapport au modèle de référence (Nelson et Siegel) sont très faibles.

En outre, Gbongué (2019) ${ }^{12}$ s'intéresse à l'actualisation de la courbe des taux zéro-coupon, en regard des mutations réglementaires. En appliquant la méthodologie décrite dans Gbongué et Planchet (2015), sur les données financières du 31/12/2017 publiées par la Bourse Régionale des Valeurs Mobilières (BRVM), il constate que le modèle de Svensson s'ajuste le mieux aux données de cette zone. Par comparaison à ces travaux antérieurs, il conclut que l'utilisation des modèles de taux d'intérêt, comprenant un nombre important de facteurs, est requise pour mieux ajuster les données de la zone UEMOA.

Aussi, Muthoni et autres (2015) proposent une courbe des taux zéro-coupon pour la Bourse de Nairobi, en comparant plusieurs modèles de taux d'intérêt. Ils constatent que le modèle de Nelson et Siegel (1987) est le plus adapté. Dans Lartey et Li (2018), les auteurs proposent un cadre méthodologique pour construire les courbes des taux zéro-coupon et forward du gouvernement ghanéen, à partir des données du marché secondaire. Ils concluent que le modèle d'interpolation par spline cubique d'Hermite s'adapte le mieux aux données du Ghana que les autres modèles de taux candidats ${ }^{13}$.

Enfin, Gbongué et autres (2017) proposent un générateur de scénarios économiques (GSE) adapté à la zone de la Conférence Interafricaine de la Prévoyance Sociale (CIPRES). Ce GSE se base sur deux variables motrices que sont les taux d'intérêt nominaux et l'indice harmonisé des prix à la consommation (IHPC). En utilisant le modèle de Nelson Siegel, ils simulent les taux d'intérêt nominaux du GSE.

De ce qui précède, cette contribution scientifique vise à améliorer la méthodologie de construction de la courbe des taux sans risque en réduisant le risque d'estimation du modèle, par comparaison aux 
travaux précédents, au regard de son intérêt grandissant pour la zone UEMOA. En clair, elle propose un nouveau modèle capable d'estimer quasiment les prix des obligations souveraines observés sur le marché secondaire (BRVM), avec des erreurs d'estimation plus faibles. Sur ce point, la méthodologie ainsi que l'implémentation sont présentées respectivement dans les sections 3 et 4 . L'avantage de ce modèle est qu'il s'appuie également sur les fondamentaux du modèle de Nelson et Siegel, ce qui permet d'interpréter ses paramètres, d'envisager la construction de la courbe des taux sans risque dynamique et d'analyser l'impact des politiques économiques dans une perspective de recherche future.

\section{MÉTHOdOLOGIE}

Cette section présente en premier lieu les principales notations et définitions. En second lieu, elle s'intéresse à la formulation du modèle de taux d'intérêt dans la zone UEMOA, sans oublier d'expliciter le processus de calibrage, ainsi que les indicateurs de mesure de la qualité d'ajustement.

\subsection{Notations et définitions}

Dans cette sous-section, nous nous intéressons à quelques notions importantes, à savoir le coefficient d'actualisation, le taux sans risque, les valeurs théoriques et observées d'une obligation souveraine, ainsi que les taux à terme. Ces informations sont utiles pour comprendre la méthodologie de construction de la courbe des taux sans risque dans la zone UEMOA.

\subsubsection{Notations}

Les principales notations de cette section sont résumées dans le tableau suivant: 


\section{TABLEAU 1 Les principales notations}

\begin{tabular}{l|l}
\multicolumn{1}{c|}{ VARIABLES } & SIGNIFICATION \\
$N, n$ & Le nombre d'obligations et de Cash-flows \\
\hline$t, T$ & Les dates de cotation et de maturité des obligations \\
\hline$\theta$ & La maturité ou durée de vie restante de l'obligation en année. $\theta=(T-t) / 365$ \\
\hline$A C_{t}^{k}$ & Les intérêts courus de l'obligation $k$ à la date de cotation $t$ \\
\hline$B_{t}(m)$ & Le coefficient d'actualisation à la date de cotation $t$ et à la maturité $m$ \\
\hline$C^{k}$ et $C^{k}$ & Le taux coupon et le coupon de l'obligation $k$ \\
\hline$C F_{t}^{k}(m)$ & Le flux généré par l'obligation $k$, à la date de cotation $t$, pour la maturité $m$ \\
\hline$F V^{k}$ & La valeur nominale de l'obligation $k$ \\
\hline$P_{c}^{k}(t, \theta)$ & Le prix côté de l'obligation $k$, à la date de cotation $t$, pour la maturité $\theta$ \\
\hline$P^{k}(t, \theta)$ & Le prix de marché de l'obligation $k$, à la date de cotation $t$, pour la maturité $\theta$ \\
\hline$\hat{P}^{k}(t, \theta)$ & Le prix théorique de l'obligation $k$, à la date de cotation $t$, pour la maturité $\theta$ \\
\hline$R_{t}(m)$ & Le taux sans risque, à la date de cotation $t$, pour la maturité $m$ \\
\hline$f_{t}(m)$ & Le taux forward instantané, à la date de cotation $t$, pour la maturité $m$ \\
\hline$D^{k}$ & La duration modifiée de l'obligation $k$ \\
\hline
\end{tabular}

\subsubsection{Définitions}

En absence d'opportunité d'arbitrage, la valeur théorique $\hat{P}^{k}(t, \theta)$ d'une obligation avec coupon est égale à la somme du produit des flux $C F_{t}^{k}(m)$ par les coefficients d'actualisation $B_{t}(m)$, pour les maturités $m$ allant de $1, \ldots,[\theta]+1$, avec $[\theta]$, la partie entière de $\theta$. Cette définition se traduit par la relation ci-dessous:

$$
\begin{aligned}
\underbrace{\hat{\boldsymbol{P}}^{k}(t, \theta)}_{\text {Valeur théorique }} & =\underbrace{P_{c}^{k}(t, \theta)+A C_{t}^{k}}_{\text {Valeur observée }} \\
& =\sum_{m=1}^{[\theta]+1} C F_{t}^{k}(m) \times B_{t}(m)
\end{aligned}
$$

Dans l'expression supra, la quantité $\hat{P}^{k}(t, \theta)=P_{c}^{k}(t, \theta)+A C_{t}^{k}$ est le prix de marché de l'obligation, observé à la date de cotation $t$. Les flux $C F_{t}^{k}$ sont versés tout le long de la durée de vie restante de l'obligation $[\theta]+1$ et respectent la relation suivante :

$$
C F_{t}^{k}(1)=\cdots=C F_{t}^{k}([\theta])=C^{k}=c^{k} \times F V^{k} \text { et } C F_{t}^{k}([\theta]+1)=C^{k}+F V^{k} \text {. }
$$


De plus, le dernier flux versé à la maturité $m=[\theta]+1$, correspond à la somme du coupon $C^{k}$ et de la valeur nominale $F V^{k}$ de l'obligation. En outre, les intérêts courus $A C_{t}^{k}$ sont calculés de la manière suivante:

$$
A C_{t}^{k}=C^{k} \times\left(\frac{t-t_{a v}}{t_{a p}-t_{a v}}\right)
$$

Avec:

$t_{a v}$ : la date de versement du coupon précédent.

$t_{a p}$ : la date de versement du coupon suivant.

Dans la littérature, il existe une relation classique entre le coefficient d'actualisation $B_{t}(m)^{14}$ et le taux sans risque $R_{t}(m)^{15}$, à la maturité $m$. Elle se définie comme suit:

$$
B_{t}(m)=\exp \left(-m \times R_{t}(m)\right) .
$$

Ce qui permet d'écrire:

$$
R_{t}(m)=-\frac{1}{m} \times \log \left(B_{t}(m)\right) .
$$

La fonction $m \mapsto R_{t}(m)$ est appelée la courbe de rendement des obligations d'État à la date de cotation $t$. Elle peut être obtenue directement à partir des taux à terme instantané $f_{t}(m)$, c'est-à-dire:

$$
R_{t}(m)=\frac{1}{m} \int_{0}^{m} f_{t}(u) d u
$$

Les taux à terme ou les taux d'intérêt "forward" sont des taux d'intérêt sur les investissements ou les prêts différés ${ }^{16}$. Nous notons par $F_{t}\left(t^{\prime}, T\right)$ le taux de rendement à la date $t$, d'un contrat à terme commençant à la date $t^{\prime}$ et finissant à la date $T$. Il se calcule à l'aide de la relation ci-dessous:

$$
F_{t}\left(t^{\prime}, T\right)=\frac{(T-t) R_{t}(T-t)-\left(t^{\prime}-t\right) R_{t}\left(t^{\prime}-t\right)}{T-t^{\prime}}
$$


En posant que: $\tau=t^{\prime}-t$ et $m=T-t^{\prime}$, nous réécrivons l'équation (3.a) sous la forme:

$$
F_{t}(\tau, m)=\frac{(\tau+m) R_{t}(\tau+m)-\tau R_{t}(\tau)}{m}
$$

Cette relation est intéressante, car elle permet d'extraire les taux à terme à partir des taux sans risque. Autrement dit, les taux à terme contiennent la même information que les taux sans risque mais sont codés de façon différente. Par conséquent, ils peuvent être considérés comme des taux à terme moyen.

\subsection{Amélioration de la méthodologie de construction de la courbe des taux sans risque dans la zone UEMOA}

\subsubsection{Modélisation}

Rappelons que le modèle proposé est une synthèse des conclusions de Gbongué et Planchet (2015) et Gbongué (2019). L'auteur retient que l'ajustement des données financières de la zone UEMOA nécessite un nombre de facteurs plus importants que le modèle de Nelson et Siegel ${ }^{17}$, afin de réduire le risque d'estimation du modèle. Dans ce contexte, le modèle de Svensson $(1994 \mathrm{~b})^{18}$ a été proposé pour cette zone au 31/12/2017, au détriment du modèle de Bjork et Christensen ${ }^{19}$ au 27/02/2015. Toutefois, nous constatons que le modèle de Nelson et Siegel est robuste et fournit aussi des résultats intéressants, en ce sens que les écarts des erreurs d'estimation des prix ne sont pas trop importants. Cependant, ils ne sont pas négligeables, ce qui suggère l'utilisation d'un modèle de taux d'intérêt contenant trois facteurs (niveau, pente et courbure), capable de fournir un risque d'estimation plus faible que les trois modèles étudiés dans Gbongué (2019).

Le modèle proposé permet d'affiner la flexibilité et la précision de la courbe des taux sans risque dans la zone UEMOA ${ }^{20}$, tout en s'appuyant sur les fondamentaux du modèle de référence Nelson et Siegel. De manière pratique, nous supposons que le taux forward instantané $f_{t}^{N E W}(m)$ suit la dynamique suivante:

$f_{t}^{N E W}(m)=\beta_{0, t}+\beta_{1, t} \exp \left(-\frac{k_{1, t}}{\tau_{1, t}} m\right)+\beta_{2, t}\left(\frac{k_{2, t}}{\tau_{2, t}} m\right) \exp \left(-\frac{k_{2, t}}{\tau_{2, t}} m\right)$ 
À partir de la relation (2.c), nous déduisons une solution analytique du taux sans risque correspondant à la maturité $m$ :

$$
\begin{aligned}
R_{t}^{N E W}(m)= & \frac{1}{m} \int_{0}^{m} f_{t}^{N E W}(u) d u \\
= & \beta_{0, t}+\beta_{1, t}\left[\frac{1-\exp \left(-\frac{k_{1, t}}{\tau_{1, t}} m\right)}{\frac{k_{1, t}}{\tau_{1, t}} m}\right] \\
& +\beta_{2, t}\left[\frac{1-\exp \left(-\frac{k_{2, t}}{\tau_{2, t}}\right)}{\frac{k_{2, t}}{\tau_{2, t}} m}-\exp \left(-\frac{k_{2, t}}{\tau_{2, t}} m\right)\right]
\end{aligned}
$$

Pour mettre en lumière les facteurs de la courbe des taux, nous réécrivons l'équation (4.b) sous la forme (cf. Gbongué [2019]):

$$
\begin{aligned}
R_{t}(m)=L_{t}-S_{t}\left[\frac{1-\exp \left(-\frac{k_{1, t}}{\tau_{1, t}}\right)}{\frac{k_{1, t}}{\tau_{1, t}} m}\right] \\
+C_{t}\left[\frac{1-\exp \left(-\frac{k_{2, t}}{\tau_{2, t}}\right)}{\frac{k_{2, t}}{\tau_{2, t}} m}-\exp \left(-\frac{k_{2, t}}{\tau_{2, t}} m\right)\right]
\end{aligned}
$$

À une date de cotation $t$, le triplet $F_{t}=\left(L_{t}, S_{t}, C_{t}\right)$ représente les facteurs de la courbe des taux sans risque ${ }^{21}$ dans la zone UEMOA. Par rapport au modèle de référence Nelson et Siegel, nous remarquons que la courbe des taux proposée dépend de sept (7) paramètres $\varsigma_{t}=\left(L_{t},-S_{t}, C_{t}, \tau_{1, t}, \tau_{2, t}, k_{1, t}, k_{2, t}\right)$ à estimer. Notons qu'il est difficile de donner une interprétation économique aux coefficients ${ }^{22} k_{1, t}$ et $k_{2, t}$. Cependant, nous constatons de manière empirique qu'ils permettent d'améliorer la précision de la courbe en réduisant les erreurs 
d'estimations. De plus, nous notons que le modèle proposé s'interprète de la même manière que le modèle de Nelson et Siegel. En effet, nous avons les relations suivantes:

$$
\forall k_{1, t}, k_{2, t} \succ 0 ; \lim _{m \rightarrow+\infty} R_{t}(m)=L_{t}, \lim _{m \rightarrow 0}\left(R_{t}(m)-L_{t}\right)=-S_{t}
$$

Pour rappel, nous avons:

$$
\begin{gathered}
\lim _{m \rightarrow 0}\left(R_{t}(m)-L_{t}\right)=-S_{t}, \\
\text { car } \lim _{m \rightarrow 0}\left[\frac{\left.1-\exp \left(-\frac{k_{1, t}}{\tau_{1, t}}\right)\right]}{\frac{k_{1, t}}{\tau_{1, t}} m}\right]=1, \\
\text { étant donné que } \lim _{\mathrm{X} \rightarrow 0} \frac{e^{X}-1}{X}=1 .
\end{gathered}
$$

L'équation (4.d) signifie que:

- Le niveau $L_{t}$ est en réalité le taux d'intérêt long terme de la courbe.

- La pente (différence entre le taux long et le taux court) est: $S_{t}$.

\subsubsection{Méthodologie de calibrage}

Cette étape est importante. En règle générale, le principe de calibrage est basé sur la minimisation de la moyenne du carré de l'erreur. Par rapport aux travaux antérieurs ${ }^{23}$, nous révisons la méthodologie de calibrage de ce modèle. En clair, nous reformulons la fonction objective $H_{1}(\hat{P})$ et proposons de résoudre le problème d'optimisation ci-dessous :

$$
\operatorname{Min}_{\varsigma} H_{1}(\hat{P})=\operatorname{Min}_{\varsigma}\left(\sum_{k=1}^{N}\left(\frac{P^{k}(t, \theta)-\hat{P}^{k}(t, \theta, \varsigma)}{D^{k}}\right)^{2}\right)
$$

Notons que les variables $D^{k}, P^{k}(t, \theta)$ et $\hat{P}^{k}(t, \theta, \varsigma)$ représentent respectivement la duration modifiée, les valeurs observées et théoriques des obligations souveraines. L'équation (5.a) requiert de déterminer 
une solution analytique de la valeur théorique de l'obligation. Cette dernière est une fonction de plusieurs paramètres s à estimer, c'est-à-dire:

$$
\begin{aligned}
& \hat{P}^{k}(t, \theta, \varsigma) \\
& =\sum_{m=1}^{[\theta]+1} C^{k} \times B_{t}(m)+100 \times B_{t}([\theta]+1)
\end{aligned}
$$

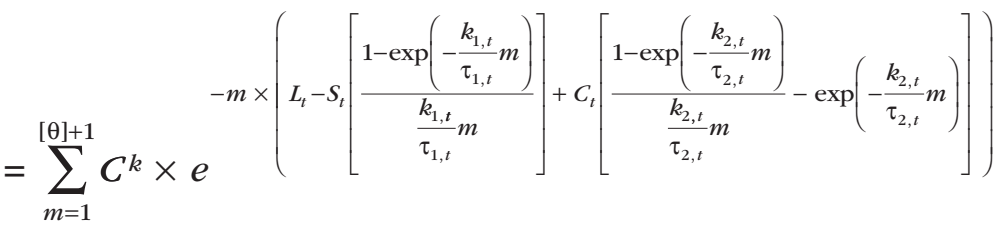

$$
\begin{aligned}
& +100 \times e \\
& -([\theta]+1) \times\left(L_{t}-S_{t}\left[\frac{1-\exp \left(-\frac{k_{1, t}}{\tau_{1, t}}[[\theta]+1)\right)}{\frac{k_{1, t}}{\tau_{1, t}}([\theta]+1)}\right]+C_{t}\left[\frac{1-\exp \left(-\frac{k_{2, t}}{\tau_{2, t}}[[\theta]+1)\right.}{\frac{k_{2, t}}{\tau_{2, t}}([\theta]+1)}-\exp \left(-\frac{k_{2, t}}{\tau_{2, t}}([\theta]+1)\right)\right]\right)
\end{aligned}
$$

La fonction prix supra est utilisée pour alimenter le problème d'optimisation (5.a), en posant des contraintes sur les paramètres du modèle, ainsi que le facteur d'actualisation. L'équation ci-dessous illustre cette assertion:

$$
\begin{array}{r}
\underset{s_{t}}{\operatorname{Min}} H_{1}(\hat{P})=\underset{s_{t}=\left(L_{t}, s_{t}, C_{t}, \tau_{1, t}, \tau_{2, t}, k_{1, t}, R_{2, t}\right)}{\operatorname{Min}}\left(\sum_{k=1}^{N}\left(\frac{P^{k}(t, \theta)-\hat{P}^{k}(t, \theta)}{D^{k}}\right)^{2}\right) \\
\text { S.C }\left\{\begin{array}{c}
U F R \leq L_{t} \leq 15 \\
-15 \leq S_{t} \leq \delta-U F R \\
-30 \leq C_{t} \leq 30 \\
\tau_{1, t}^{N S} \leq \tau_{1, t} \leq 30 \\
0 \leq \tau_{2, t} \leq 30 \\
1 \leq k_{1, t} \leq 10 \\
1 \leq k_{2, t} \leq 10 \\
B_{t}(0)=1 \\
B_{t}(100)=0
\end{array}\right.
\end{array}
$$


Nous proposons une procédure simple, afin de résoudre ce problème d'optimisation à la date de cotation $t$. En amont, elle requiert de déterminer les paramètres du modèle de Nelson et Siegel. En aval, il s'agira de suivre les étapes suivantes, en respectant les contraintes issues de l'équation (5.c):

Étape 1 : Fixer les paramètres $\left(k_{1, t}, k_{2, t}, \tau_{1, t}\right)$. Notons que le choix initial de $\tau_{1, t}$ est $\tau_{1, t}^{N S}$.

Étape 2 : Déterminer les paramètres $\left(L_{t},-S_{t}, C_{t}\right)$ pour les valeurs de $\tau_{2, t}$ compris entre 0 et 30 , en résolvant le problème d'optimisation $H_{1}(\hat{P})$.

Étape 3a: Retenir les paramètres optimaux $\left(L_{t}^{\text {opt }},-S_{t}^{o p t}, C_{t}^{o p t}\right)$, qui respectent les conditions $L_{t}^{o p t} \succ 0$ et $L_{t}^{o p t}-S_{t}^{o p t} \succ 0$, pour la valeur la plus faible de $H_{1}(\hat{P})$ et déduire la valeur de $\tau_{2, t}$ correspondante.

Étape 3b: Calculer les erreurs d'estimation en l'occurrence la MAPE, le Theil-U-Statistic et le coefficient de variation ${ }^{24}$.

Étape 4: Répéter plusieurs fois les étapes 1 à 3, en faisant varier la valeur des paramètres $k_{1, t}, k_{2, t}$ et $\tau_{1, t}$, sur les intervalles spécifiés dans le problème d'optimisation, tout en conservant les erreurs d'estimation issues de l'étape 3.

Étape 5: Comparer les erreurs d'estimation de l'étape précédente. Nous retenons les paramètres optimaux $\mathrm{s}_{t}^{o p t}=\left(L_{t}^{o p t},-S_{t}^{o p t}, C_{t}^{o p t}, \tau_{1, t}^{o p t}, \tau_{2, t}^{o p t}, k_{1, t}^{o p t}, k_{2, t}^{o p t}\right)$ qui présentent le risque d'estimation (MAPE, Theil-U-Statistic) le plus faible.

\subsubsection{Mesure du risque d'estimation}

Dans la littérature, la comparaison des modèles de construction d'une courbe des taux sans risque peut se faire selon deux approches, à savoir: l'approche graphique ( $c f$. Stander [2005]) et l'approche quantitative par le calcul des indicateurs de dispersion (cf. Brousseau [2002], Anderson et Sleath [2001], Aljinović et autres [2012], Kovachev et Simeonov [2014], Bolder et Streliski [1999], Navas [2005], Hladíková et Radová [2012], Martellini et autres [2005]).

Nous nous intéressons à la seconde approche. Dans ce contexte, nous notons que Kovachev et Simeonov (2014) utilisent trois indicateurs pour évaluer la qualité d'ajustement des modèles de Svensson et VRP (Variable Roughness Penalty). Ces indicateurs sont: Mean squared error (MSE), Root mean squared error (RMSE) et Theil-U-statistic. Par contre, Navas (2005) compare les modèles de Chan et autres (1992), 
Schaefer et Schwartz (1984), Cox, Ingersoll, et Ross (1985), en utilisant le Mean absolute percentage error (MAPE) et le coefficient de détermination $R^{2}$. Les indicateurs retenus dans cette sous-sous-section sont résumés dans le tableau ci-dessous:

\section{TABLEAU 2 Les indicateurs de mesure de la qualité du modèle de taux d'intérêt}

\begin{tabular}{l|l}
\multicolumn{1}{c|}{ INDICATEURS } & EXPRESSION \\
Theil-U-statistic & $U=\frac{\sqrt{\sum_{k=1}^{N} \frac{\left(P^{k}(t, \theta)-\hat{P}^{k}(t, \theta)\right)^{2}}{N}}}{\sqrt{\sum_{k=1}^{N} \frac{\left(\hat{P}^{k}(t, \theta)\right)^{2}}{N}}+\sqrt{\sum_{k=1}^{N} \frac{\left(P^{k}(t, \theta)\right)^{2}}{N}}}$ \\
\hline Mean absolute percentage error & MAPE $=\frac{1}{N} \sum_{k=1}^{N} \frac{\left|P^{k}(t, \theta)-\hat{P}^{k}(t, \theta)\right|}{P^{k}(t, \theta)}$ \\
\hline Coefficient de variation & $\mathrm{CV}=\frac{\sigma\left(X_{1}, \cdots X_{N}\right)}{E\left(X_{1}, \cdots X_{N}\right)}$ \\
& $\forall k=1, \ldots, N ; X_{k}=\left[P^{k}(t, \theta)-\hat{P}^{k}(t, \theta)\right]^{2}$
\end{tabular}

Par référence aux travaux précédents, nous retenons aussi le MAPE et le Theil-U-Statistic, pour mesurer la qualité d'ajustement du modèle des taux d'intérêt de la zone UEMOA. En pratique, nous optons pour ces indicateurs de qualité, car ils sont adaptés à l'approche par les prix des obligations retenue dans cet article. Cependant, le coefficient de variation $\mathrm{CV}$ sera considéré comme un indicateur de robustesse.

\section{ANALYSE EMPIRIQUE}

Dans cette section, nous nous intéressons d'abord à la détermination de la structure par terme des taux d'intérêt (données, résultats et analyse de sensibilité). Ensuite, nous analysons statistiquement les variations des rendements obligataires, ainsi que les facteurs de la courbe des taux sans risque sur la période 2012-2017. Enfin, nous discutons les résultats obtenus. 


\subsection{Structures par terme des taux d'intérêt dans la zone UEMOA}

\subsubsection{Données utilisées}

Les données utilisées sont mensuelles et couvrent la période du 31/01/2012 au 31/12/2017. À chaque fin de mois (ou date de cotation $t$ ), la base de données finale servant de «input» à la modélisation de la courbe des taux sans risque comprend les champs suivants:

- Le code des obligations

- Les dates de cotation, d'émission ${ }^{25}$, de jouissance ${ }^{26}$ et de maturité27.

- Les prix cotés et de marchéer de l'obligation

- Le taux coupon

- Les intérêts courus

- La durée de vie résiduelle de l'obligation ${ }^{29}$.

- La maturité de l'obligation (en année) ${ }^{30}$.

Au 31/12/2017, nous avons 18 obligations en circulation à la BRVM, comprenant les émetteurs suivants : Côte d'Ivoire (9), Sénégal (4), Mali (2), Burkina Faso (2) et Bénin (1). Les statistiques descriptives sont résumées dans le tableau ci-dessous:

TABLEAU 3 Statistiques descriptives des données financières observées au $31 / 12 / 2017$

\begin{tabular}{l|c|c|c|c|c|c}
\multicolumn{2}{c}{ MIN } & \multicolumn{2}{c}{ MAX } & \multicolumn{2}{c}{ 1ER QUANTILE } & \multicolumn{2}{c}{ MÉDIANE } & \multicolumn{2}{c}{ MOYENNE } & 3E QUANTILE \\
\hline Prix de marché & 100,2 & 105 & 100,6 & 102,1 & 102,3 & 104,1 \\
\hline Coupon (\%) & 5,85 & 6,70 & 5,99 & 6,30 & 6,27 & 6,50 \\
\hline Durée de vie résiduelle & 0,93 & 11,26 & 3,66 & 5,95 & 6,23 & 8,78 \\
\hline Maturité & 5 & 12 & 7 & 8 & 8,44 & 10
\end{tabular}




\subsubsection{Résultats au $31 / 12 / 2017$}

À partir des données du 31/12/2017, nous construisons la courbe des taux sans risque de référence de la zone $\mathrm{UEMOA}^{31}$, à partir du modèle décrit ci-dessus. Pour ce faire, nous formulons les hypothèses suivantes :

- Les émetteurs sont de qualité similaire, ce qui justifie l'estimation de la valeur théorique des obligations souveraines par l'équation 1.a.

- $U F R=6,2 \%^{32}$, ce qui permet de contrôler le taux long terme.

- $\delta=2,5 \%^{33}$. Il s'agit du taux directeur ou taux minimum de soumission fixé par la Banque Centrale des États de l'Afrique de l'Ouest (BCEAO) de la zone UEMOA, qui correspond au taux d'intérêt de maturité nulle $(\mathrm{m}=0)$.

L'application de la procédure de calibrage permet d'obtenir les paramètres du modèle proposé. De plus, nous estimons les paramètres des modèles de Nelson et Siegel (1987), Svensson (1994) et Björk et Christensen (1999), que nous résumons dans le tableau 4 ci-dessous:

TABLEAU 4 Estimation des paramètres des modèles de taux d'intérêt au $31 / 12 / 2017$

\begin{tabular}{|c|c|c|c|c|}
\hline $\begin{array}{l}\text { PARAMĖTRES } \\
\text { À } T=31 / 12 / 2017\end{array}$ & $\begin{array}{l}\text { NELSON SIEGEL } \\
\text { (EN POURCENTAGE) }\end{array}$ & $\begin{array}{c}\text { SVENSSON } \\
\text { (EN POURCENTAGE) }\end{array}$ & $\begin{array}{c}\text { BJÖRK } \\
\text { ET CHRISTENSEN } \\
\text { (EN POURCENTAGE) }\end{array}$ & $\begin{array}{l}\text { MODĖLE PROPOSÉ } \\
\text { (EN POURCENTAGE) }\end{array}$ \\
\hline$L_{t}$ & 6,2 & 6,2 & 6,2 & 6,2 \\
\hline$-S_{t}$ ou $-S_{1, t}$ & $-3,7$ & $-3,7$ & $-1,85$ & $-3,7$ \\
\hline$C_{t}$ ou $C_{1, t}$ & 0,15 & 6,22 & $-0,564$ & $-2,096$ \\
\hline$C_{2, t}$ ou $-S_{2, t}$ & Non Disponible & $-3,7$ & $-1,85$ & Non Disponible \\
\hline$\tau_{1, t}$ & 0,9 & 1,1 & 1,2 & 0,8 \\
\hline$\tau_{2, t}$ & Non Disponible & 2,8 & Non Disponible & 4 \\
\hline$k_{1, t}$ & Non Disponible & Non Disponible & Non Disponible & 3 \\
\hline$k_{2, t}$ & Non Disponible & Non Disponible & Non Disponible & 2 \\
\hline
\end{tabular}

À partir de l'estimation des paramètres, nous pouvons représenter graphiquement les courbes de taux sans risque provenant de ces quatre modèles, à la date de cotation du 31/12/2017. La figure 1 révèle que 
les courbes des taux sans risque obtenues sont croissantes, avec des pentes positives. Ce constat est naturellement cohérent avec les performances économiques actuelles ${ }^{34}$ de la zone UEMOA:

FIGURE 1 Évolution des courbes des taux ZC de l'UMOA au 31/12/2017 issues de plusieurs modèles

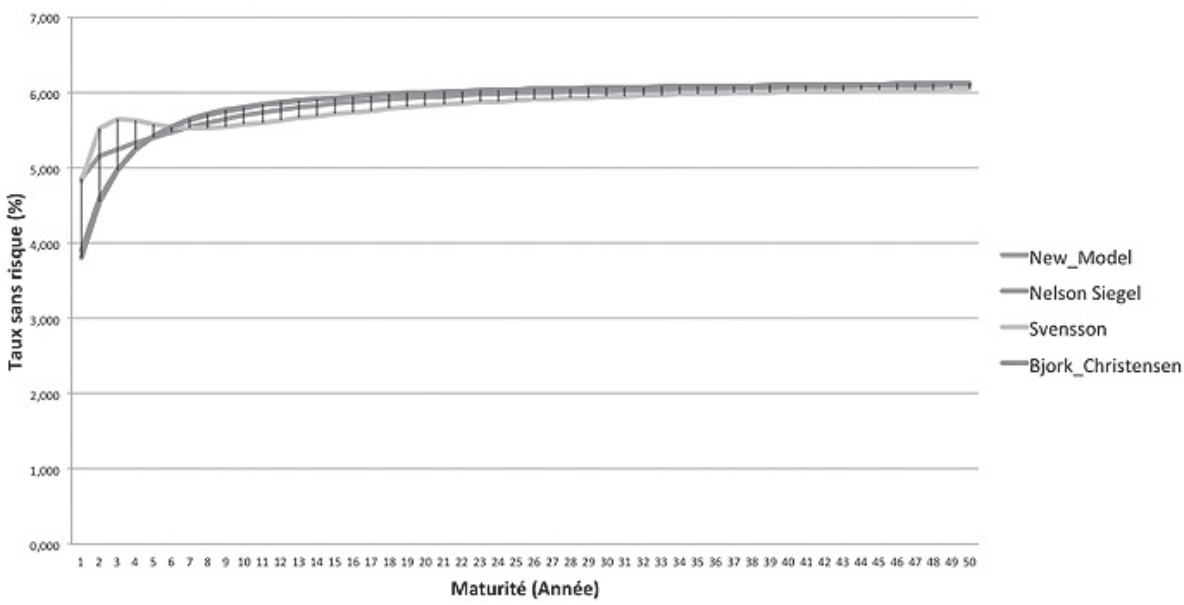

Pour mesurer le risque d'estimation de ces modèles, nous calculons le Theil-U-Statistic et le Mean Absolute Percentage Error (MAPE). Nous pouvons conclure que le modèle de taux d'intérêt proposé s'ajuste le mieux aux données de l'UEMOA à la date de cotation du 31/12/2017, comme l'illustre le tableau 5:

TABLEAU 5 Détermination des erreurs d'estimation des prix au 31/12/2017 sous plusieurs modèles

\begin{tabular}{l|c|c|c|c}
\multicolumn{4}{c}{$\begin{array}{c}\text { MODËLE PROPOSÉ } \\
\text { (EN POURCENTAGE) }\end{array}$} & $\begin{array}{c}\text { NELSON ET SIEGEL } \\
\text { (EN POURCENTAGE) }\end{array}$ \\
$\begin{array}{l}\text { STATISTIQUES } \\
\text { (EN POURCENTAGE) }\end{array}$ & $\begin{array}{c}\text { BJÖRK } \\
\text { (EN PHRISTENSEN }\end{array}$ \\
Theil U-Statistic & 1,14 & 1,296 & 1,15 & 1,28 \\
\hline MAPE & 1,87 & 2,116 & 1,89 & 2,1 \\
\hline $\begin{array}{l}\text { Coefficient } \\
\text { de variation }\end{array}$ & 1,12 & 1,17 & 1,15 & 1,16
\end{tabular}


Afin d'illustrer la pertinence de ce modèle $(N W)$, nous proposons de calculer deux indicateurs qui s'apparentent à la définition de la précision d'une mesure quelconque ( $c f$. Molenaars et autres [2015]):

$$
\begin{gathered}
E_{1}=\frac{U_{N W}-U_{\text {Model }}}{U_{N W}} \\
E_{2}=\frac{M A P E_{N W}-M A P E_{\text {Model }}}{M A P E_{N W}} \\
E_{3}=\frac{C V_{N W}-C V_{\text {Model }}}{C V_{N W}}
\end{gathered}
$$

Ces indicateurs (E1, E2 et E3) révèlent que le modèle proposé s'ajuste le mieux aux données de l'UEMOA au 31/12/2017 et permet globalement de réduire le risque d'estimation par comparaison aux modèles de Nelson et Siegel, Svensson et Björk et Christensen. Autrement dit, il permet de retrouver quasiment les prix des obligations souveraines observées sur le marché financier secondaire de la zone UEMOA. Par conséquent, il est adapté aux données de cette zone et permet d'obtenir une meilleure estimation des taux sans risque, en accord avec la réalité économique. Le tableau 6 ci-dessous illustre cette assertion:

\begin{tabular}{|c|c|c|c|c|c|c|c|}
\hline STATISTIQUES & $\begin{array}{l}\text { MODĖLE } \\
\text { PROPOSÉ }\end{array}$ & $\begin{array}{l}\text { NELSON } \\
\text { ET SIEGEL }\end{array}$ & $\begin{array}{l}\text { E1, E2, E3 } \\
\text { (NW-NS) }\end{array}$ & SVENSSON & $\begin{array}{l}\text { E1, E2, E3 } \\
\text { (NW-SV) }\end{array}$ & $\begin{array}{c}\text { BJÖRK ET } \\
\text { CHRISTENSEN }\end{array}$ & $\begin{array}{c}\text { E1, E2, E3 } \\
\text { (NW-SV) }\end{array}$ \\
\hline Theil U-Statistic & 1,14 & 1,296 & $-14 \%$ & 1,15 & $-1 \%$ & 1,28 & $-12 \%$ \\
\hline MAPE & 1,87 & 2,116 & $-13 \%$ & 1,89 & $-1 \%$ & 2,1 & $-12 \%$ \\
\hline $\begin{array}{l}\text { Coefficient } \\
\text { de variation }\end{array}$ & 1,12 & 1,17 & $-4 \%$ & 1,15 & $-3 \%$ & 1,16 & $-4 \%$ \\
\hline
\end{tabular}

\section{TABLEAU 6 Variation des erreurs d'estimation des prix du nouveau modèle par rapport aux modèles candidats}

En nous basant sur les équations (3.b), (4.a) et des paramètres issus du tableau 4, nous déterminons les courbes des taux forward $F_{t}(\tau, 0)$ et $F_{t}(\tau, 1)^{35}$ au $31 / 12 / 2017$. Nous observons qu'elles se situent audessus de la courbe des taux sans risque, ce qui est conforme aux attentes théoriques ${ }^{36}$. Aussi, nous observons sur la figure 2 que les taux forward convergent plus vite que les taux sans risque vers l'UFR (6,2\%), avec une vitesse de convergence égale à la maturité 16 ans. Notons que le dernier point liquide (Last liquid point) correspond à la maturité 7 ans. Autrement dit, la liquidité des obligations souveraines devient moins importante au-delà de cette maturité. Cette situation peut 
expliquer, a priori, la difficulté des émetteurs souverains à mobiliser des ressources importantes sur le long terme, ce qui impacte la stratégie de gestion de la dette publique à moyen et long terme.

FIGURE 2 Analyse comparative des courbes des taux sans risque et forward au 31/12/2017

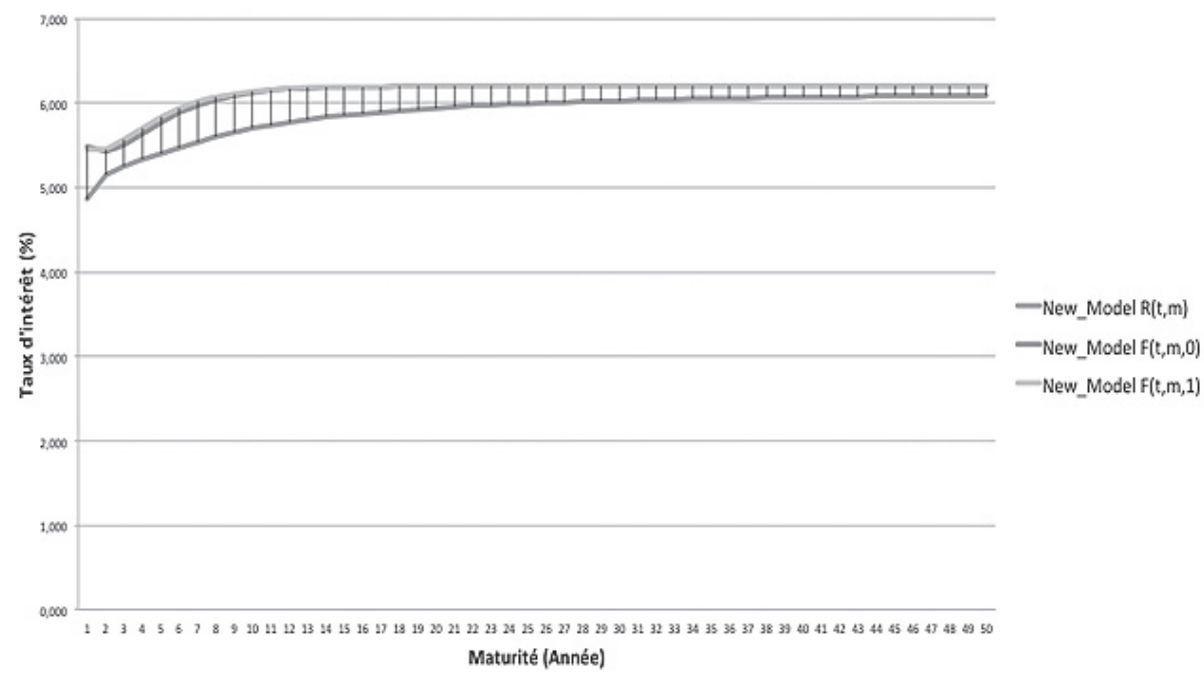

\subsubsection{Analyse de sensibilité}

Gbongué (2019) montre que la courbe des taux sans risque est sensible à ses hypothèses de construction pour les modèles de type Nelson et Siegel. Ce résultat se vérifie naturellement dans le modèle proposé, étant donné qu'il s'appuie sur les fondamentaux du modèle de Nelson et Siegel. En effet, nous observons que:

- La modification des hypothèses économiques et financières (UFR et $\delta$ ) provoque un déplacement parallèle de cette courbe vers le haut ou le bas,

- La forme de la courbe des taux est influencée par le modèle de taux utilisé et la structure des données observée à la date de cotation choisie,

- La courbe des taux se déforme, peu importe le modèle de taux utilisé, si nous modifions les bornes des paramètres dans le processus de calibrage. 
En ce qui concerne les facteurs de la courbe des taux, nous remarquons qu'un choc positif du facteur niveau $L_{t}$ ou $\beta_{0, t}$ provoque naturellement un déplacement parallèle de la courbe des taux initiale vers le haut, comme l'illustre la figure 3:

FIGURE 3 Déplacement de la courbe des taux sans risque initiale suite à un choc positif du facteur niveau dans la zone UEMOA

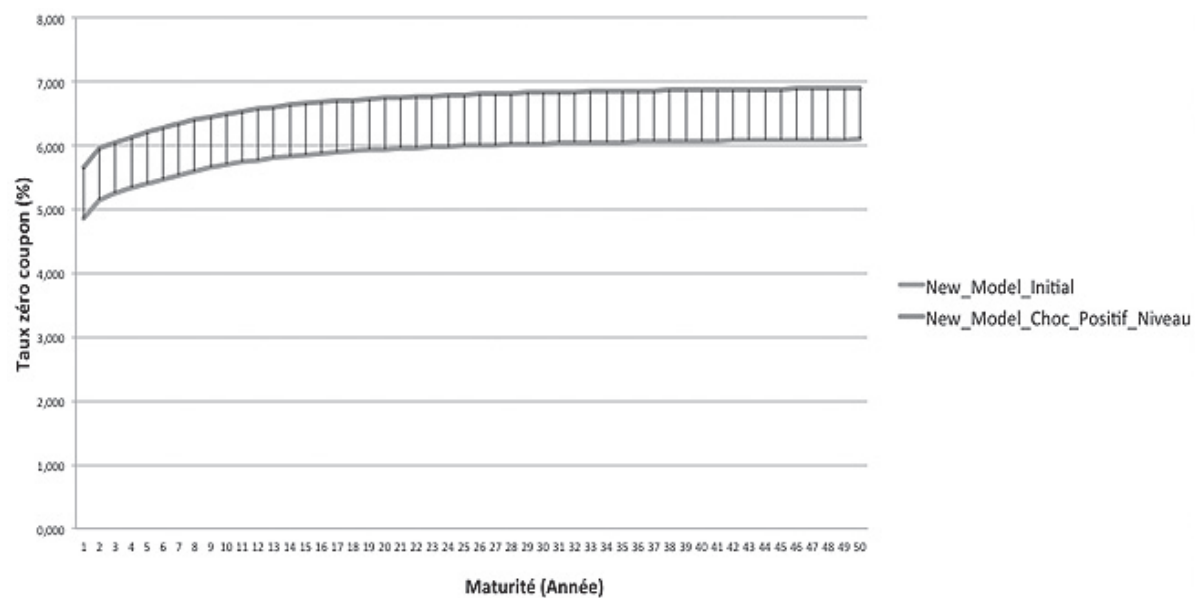

En outre, un choc positif du facteur pente $S_{t}$ ou $-\beta_{1, t}$ (par exemple à la suite d'un assouplissement des conditions monétaires dans la zone UEMOA) provoque plutôt une baisse des taux à court terme, puis une augmentation des taux longs, comme illustré dans Litterman et Scheinkman (1991). Enfin, la courbe des taux initiale se réduit souvent sur le très court et long terme, mais croît sur le moyen terme, comme suite au choc positif du facteur courbure $\beta_{2, t}$. Toutefois, ce dernier résultat reste mitigé.

\subsection{4. Évolution de la courbe des taux sans risque à plusieurs dates de cotation}

Si l'objectif de cet article n'est pas de construire une courbe des taux dynamique, en revanche, nous appliquons la méthodologie proposée sur les données financières du 31/12/2017, 31/12/2018 et 18/10/2019, afin d'étudier l'évolution de cette courbe à plusieurs dates de cotation. Nous observons que le modèle proposé est acceptable en ce sens qu'il fournit des courbes des taux stables à différentes dates. Cette assertion est illustrée par la figure 4 ci-dessous: 
FIGURE 4 Évolution de la courbe des taux sans risque à plusieurs dates de cotation dans le modèle proposé.

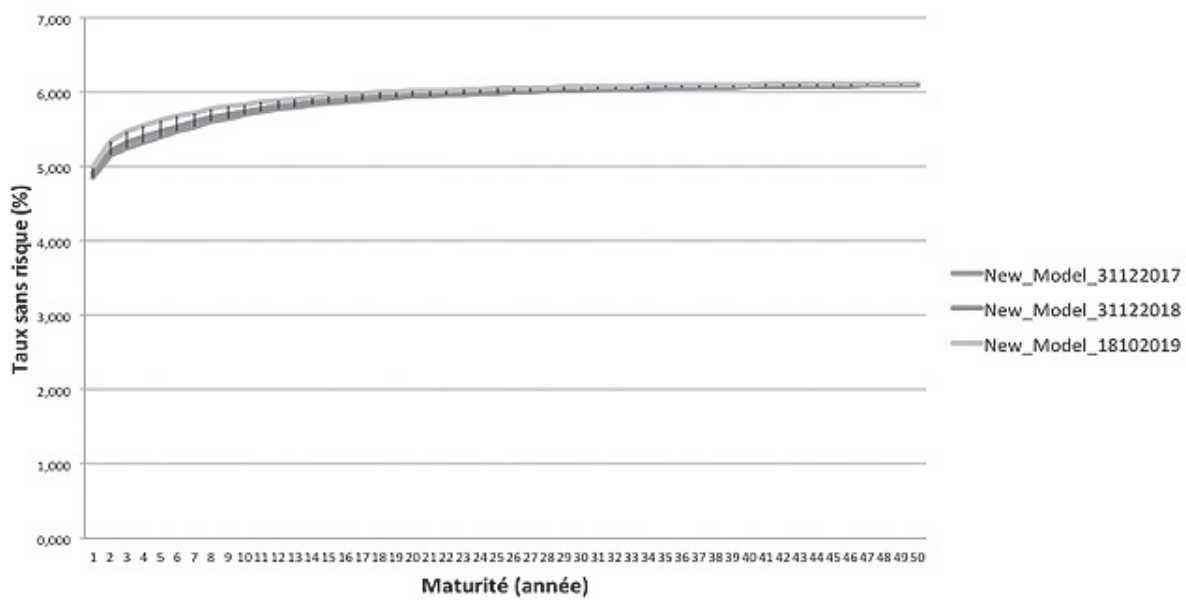

Nous remarquons que la courbe des taux sans risque se déplace vers le haut. Cette situation révèle une augmentation progressive des taux d'intérêt sur le marché financier régional de l'UEMOA, en accord avec la réalité actuelle.

\subsection{Analyse de la dynamique des courbes des taux sans risque mensuelles de l'UEMOA sur la période 2012-2017}

L'application de la méthodologie améliorée sur les données mensuelles, couvrant la période du 31/01/2012 au 31/12/2017, permet de constituer une base de données historique sur les courbes de rendement mensuel des obligations souveraines de la zone UEMOA. Notons que l'historique des facteurs de la courbe des taux sans risque est une information importante dans l'analyse des politiques économiques ${ }^{37}$. Toutefois, nous nous intéressons, dans cette sous-section, à l'analyse statistique des courbes de rendement et l'efficience du marché obligataire dans la zone UEMOA.

\subsubsection{Analyse statistique des courbes de rendement}

L'analyse statistique des données historiques mensuelles de la courbe des taux sans risque révèle que les taux à 1 an ont progressé de 2,24\%, tandis que les taux à 2, 3, 5, 7 et 10 ans ont reculé respectivement de 
$0,38 \%, 4 \%, 7,8 \%, 9,5 \%$ et $10,6 \%$ sur la période $2012-2017$. De plus, les variations mensuelles de ces taux ne suivent pas la loi normale, excepté celle de 2 ans, ce qui implique la non-normalité des rendements obligataires à 1, 3, 5, 7 et 10 ans, comme le montre la figure 5 . Cette situation remet en cause l'hypothèse de normalité des rendements, classiquement utilisée dans la gestion des risques et l'analyse quantitative. En clair, tout modèle fondé sur l'hypothèse de normalité des rendements pourrait produire des mesures inexactes des prix ou du risque dans la zone UEMOA.

L'analyse en composante principale des rendements obligataires sur la période 2012-2017 montre que la courbe des taux sans risque est gouvernée par trois facteurs (niveau, pente, courbure), ce qui est conforme aux attentes théoriques. Ces facteurs expliquent $99,7 \%$ de la variance, qui se décompose comme suit: $51,3 \%$ pour le $1^{\text {er }}$ facteur (niveau), $36,7 \%$ pour le $2^{\mathrm{e}}$ facteur (pente) et $11,7 \%$ pour le $3^{\mathrm{e}}$ facteur (courbure). La figure 6 illustre l'évolution de ces facteurs sur les maturités observables (1, 2, 3, 5, 7 et 10 ans).

\subsubsection{Analyse de l'efficience du marché obligataire de l'UEMOA}

En finance, l'efficience d'un marché suppose, de manière classique, une meilleure transmission de l'information disponible dans les prix. Dans ce cas, l'écart entre les prix des obligations observés sur le marché et les prix théoriques issus du modèle de calcul de la courbe de rendement devrait se réduire au cours du temps. Par conséquent, le comportement des erreurs d'estimation des obligations souveraines peut être interprété comme une première mesure d'appréciation de l'efficience du marché obligataire. En clair, nous pouvons dire que le marché des obligations de l'UEMOA est efficient a priori si les erreurs d'estimation des prix diminuent au fil du temps.

L'analyse statistique des erreurs d'estimation révèle qu'elles ont progressé de $37,4 \%$ et $45,2 \%$, respectivement pour la Theil-U-Statistic et la MAPE, sur la période 2012-2017. Aussi, elles ne suivent pas la loi normale $^{38}$, comme le montre la figure 7 . De plus, elles sont volatiles ${ }^{39}$, ce qui signifie que les rendements obligataires souverains observés sur le marché financier régional ne concordent pas avec ceux prévus par une courbe des taux sans risque théorique. Cette situation montre, de manière empirique, que le marché obligataire de l'UEMOA n'est pas efficient, comme l'illustre la figure 8. L'absence officielle d'une courbe des taux sans risque dans cette zone ne favorise pas le développement de son marché obligataire. Toutefois, elle constitue un préalable à un meilleur pilotage des risques liés à la dette publique (cf. Hubig [2012]). 


\subsection{Discussion}

La figure 1 montre que la courbe des taux sans risque issue du modèle proposé se situe entre les courbes des taux provenant des modèles de Nelson et Siegel, Svensson et Björk et Christensen. Ce constat révèle que ce modèle est pertinent, car il fournit des taux sans risque qui résument l'information contenue dans les autres modèles. Autrement dit, ils peuvent être interprétés comme une combinaison linéaire des taux issus des modèles candidats. De plus, ces courbes sont croissantes, avec des pentes positives, ce qui est en accord ${ }^{40}$ avec la théorie des anticipations de la structure par terme des taux d'intérêt (cf. Hicks [1939]).

Nous pouvons déduire que la forme de la courbe des taux issue du modèle proposé reflète les performances économiques actuelles, en excluant tout risque de récession économique sur le court terme. Toutefois, cette situation économique favorable risque de s'inverser sur le long terme, si le spread $^{41}$ de la courbe des taux continue de se réduire au cours des années futures, en ce sens qu'il a baissé ${ }^{42}$ de $29,8 \%$ sur la période $2012-2017$. Enfin, nous recommandons la construction d'une courbe des taux sans risque de référence pour les émissions souveraines à partir du modèle proposé, afin d'améliorer l'efficience du marché obligataire dans la zone UEMOA. Cette pratique pourrait participer à la dynamique du marché secondaire à travers la valorisation des instruments financiers, la transparence et l'élargissement de la base des investisseurs.

\section{CONCLUSION}

Nous nous sommes intéressés à l'amélioration de la méthodologie de construction de la courbe des taux sans risque, proposée dans les travaux antérieurs. De manière pratique, nous avons présenté un autre modèle de taux d'intérêt, décrit le processus de calibrage et de mesure du risque d'estimation, afin de répondre aux enjeux liés aux mutations observées dans la zone UEMOA.

L'analyse comparative révèle que ce modèle présente un risque d'estimation plus faible de l'ordre de $1,14 \%$ et $1,87 \%$, respectivement pour le Theil-U-Statistic et le MAPE, par comparaison aux modèles candidats (Nelson et Siegel, Svensson et Björk et Christensen). De plus, les résultats obtenus sont cohérents et peuvent être utilisés pour valoriser les actifs financiers sur le marché secondaire de la zone UEMOA. 
Notons que les hypothèses de construction de la courbe des taux doivent être choisies minutieusement par l'expert, au risque de disposer d'une courbe des taux qui s'écarte de la réalité. Cette situation pourrait engendrer des distorsions sur le marché.

L'application de ce modèle sur les données mensuelles, couvrant la période du 31/01/2012 au 31/12/2017, a permis de constituer une base de données importante sur la courbe de rendement des obligations souveraines, ainsi que ses facteurs. L'exploitation de ces données a permis de conclure que l'inefficience du marché obligataire retarde son développement. Une des causes est l'absence d'une courbe des taux sans risque officielle. Cette dernière doit être construite, car elle contribuera à améliorer l'efficience du marché financier et l'efficacité de la transmission monétaire, participant ainsi au développement de la zone UEMOA.

Enfin, nous pourrons envisager des perspectives de recherche grâce aux données collectées. Elles porteront principalement sur l'élaboration de la version dynamique de la courbe des taux sans risque ( $c f$. Diebold et Li [2006]), sur l'analyse de l'impact des politiques ${ }^{43}$, sur les rendements obligataires et les économies de l'union, sans oublier le développement des modèles de stratégie de la dette publique.

\section{BIBLIOGRAPHIE}

[1] AFMI (2016) "Le Guide des courbes des taux en Afrique», Document de travail, BAD.

[2] Agence UMOA-Titres (2018) «Le marché des titres publics de l'UEMOA - Acteurs et Fonctionnement", Août 2018.

[3] Ahlgrim K.C., D’Arcy S.P., Gorvett R.W. (2005) «Modeling Financial Scenarios - A Framework for the Actuarial Profession", Proceedings of the Casualty Actuarial Society, 177-238. http://www.casact.org/pubs/ proceed/proceed05/05187.pdf

[4] Aljinović Z., Poklepović T., Katalinić K. (2012) «Best fit model for yield curve estimation", Croatian Operational Research Review (CRORR), Vol. 3, 2012 .

[5] Allouche J. (2013) «La courbe des taux», BSI Economics.

[6] Andersen, N. (2018) «Yield curve modelling and a conceptual framework for estimating yield curves: evidence from the European Central Bank's yield curves", ECB Statistics Paper Series № 27. 
[7] Anderson N., Sleath J. (2001) «New estimates of the UK real and nominal yield curves", Bank of England, ISSN 1368-5562.

[8] Anderson, Sleath (2001) "New estimates of the UK real and nominal yield curves", Working Paper, Bank of England.

[9] Antonio D., Roseburgh D. (2010) «Fitting the Yield Curve Cubic spline interpolation and smooth extrapolation", Barrie + Hilbert Calibration.

[10] Bank for International Settlements (2005) «Zero-coupon yield curves: technical documentation", BIS Papers no 25.

[11] Banque Africaine de Développement (2010) "Guide des marchés obligataires africains et des produits dérivés", Document de travail, BAD.

[12] Barrett, W. R., Gosnell, T. F. Jr., Heuson, A. J., (1995) "Yield Curve Shifts and the Selection of Immunization Strategies", Journal of Fixed Income, Vol. 5, No 2, 53-64.

[13] Bliss R. (1997) "Movements in the term structure of interest rate", Economic Review, Federal Reserve Bank of Atlanta, Vol. 82, 16-33.

[14] Bliss, R., and Fama, E., (1987) "The Information in Long-Maturity Forward Rates", American Economic Review, Vol. 77, 680-692.

[15] Bolder D., Stréliski D. (1999) «Yield Curve Modelling at the Bank of Canada", Rapport technique $n^{\circ} 84$.

[16] Brousseau V. (2002) «The functional form of yield curves", European Central Bank, Working Paper no 80.

[17] Byrd, R. H., Lu, P., Nocedal, J. and Zhu, C. (1995) «A limited memory algorithm for bound constrained optimization", SIAM J. Scientific Computing, Vol. 16, p. 1190-1208.

[18] Cairns A.J.G. (1997) «Descriptive Bond-Yield and Forward-Rate models for the British government securities market ", Institute of actuaries.

[19] Carriere J. F. (1998) «Withdrawal benefits under a dependent double decrement model ", Astin bulletin. Vol. 28. № 1, pp. 49-57.

[20] CEIOPS (2010). "Qis 5 risk-free interest rates extrapolation method", Technical report, CEIOPS.

[21] Chan K.C., Karolyi G.A., Longstaff F.A., Sanders A. (1992) «An empirical comparison of alternative models of the short-term interest rate", Journal of Finance 47(3), 1209-1227.

[22] Choudhry M. (2004) "Analysing and interpreting yield curve", JOHN Wiley \& Sons 
[23] Christensen J.H.E. (2015) «A Regime-Switching Model of the Yield Curve at the Zero Bound", Federal Reserve Bank of San Francisco, Working Paper 2013-34.

[24] Christensen J.H.E., Diebold F.X., Rudebush G.D. (2010) «The Affine Arbitrage-Free Class of Nelson-Siegel Term Structure Models ", Federal Reserve Bank of San Francisco, WP no 2007-20.

[25] Coroneo, L., Nyholm, K., Vivada-Koleva, R., (2008) «How ArbitrageFree Is the Nelson-Siegel Model? „, European Central Bank, Working Paper Series, No 874.

[26] Cox J.C., Ingersoll J.E., Ross S.A. (1985) «A theory of the term structure of interest rates", Econometrica 53, 385-407.

[27] Deacon M., Derry A., (1994) «Deriving Estimates of Inflation Expectations from the Prices of UK Government Bonds", Bank of England, Working Paper No.23.

[28] Dhaene J., Tsanakas A., Valdez E.A, Vanduffel S. (2012) «Optimal capital allocation principle», Journal of Risk and Insurance, Vol. 79 (1), P.1-28.

[29] Diebold F.X., Li C. (2006) «Forecasting the term structure of government bond yields", Journal of Econometrics, Elsevier, Vol. 130 (2), p. 337-364.

[30] Diebold F.X., Rudebusch G.D., Aruoba B., (2006) «The Macroeconomy and the Yield Curve: A Dynamic Latent Factor Approach", Journal of Econometrics, Vol. 131, p. 309-338.

[31] Dobbie, G.M., Wilkie, A.D. (1978) «The Financial Times-Actuaries Fixed Interest Indices", Journal of the Institute of Actuaries, Vol. 105, p. 15-26.

[32] Dobbie, G.M., Wilkie, A.D. (1979) «The Financial Times-Actuaries Fixed Interest Indices", Transaction of the Faculty of Actuaries, Vol. 36, p.203-213.

[33] Dullmann K., Uhrig-Homburg M., Windfuhr M., (2000) «Risk structure of interest rates: an empirical analysis for deutschmark-denominated bonds", European Financial Management, Vol. 6, № 3, 367-388.

[34] European Central Bank (2008) "Euro area yield curves modeling", The ECB's directorate general statistics releases euro area yield curves every target. https://www.ecb.europa.eu/stats/financial_markets_and_ interest_rates/euro_area_yield_curves/html/index.en.html 
[35] Fabozzi, F. J., Martellini, L., Priaulet, P., (2005) "Predictability in the Shape of the Term Structure of Interest Rates", Journal of Fixed Income, Vol. 15, No 1, 40-53.

[36] Finanstilsynet (2010) "A Technical Note on the Smith-Wilson Method»

[37] Finma (2012) "Assouplissements temporaires du Test Suisse de Solvabilité SST", Rapport explicatif.

[38] Fisher, M., Nychka, D., Zervos D., (1995) «Fitting the Term Structure of Interest Rates with Smoothing Splines", Working Paper 95-1, Finance and Economics Discussion Series.

[39] Frankel, J.A., Lown, C.S. (1994) "An indicator of future inflation extracted from the steepness of the interest rate yield curve along its entirely length", The Quarterly Journal of Economics, Vol. 109, p. 339-348.

[40] Gbongué F. (2019) «Modélisation et prévision de la courbe des taux zéro-coupon pour le développement de la zone UEMOA», Revue Economique et Monétaire, № 25.

[41] Gbongué F., Planchet F. (2015) "Analyse comparative des modèles de construction d'une courbe de taux sans risque dans la zone CIPRES ", Bulletin Français d'Actuariat, Vol. 15 no 30, p 129-168.

[42] Gbongué, F., Planchet, F., Ahoussi, A. (2017) «Proposition d'un modèle de projection des scénarios économiques pour le développement de la zone CIPRES", Assurances et gestion des risques, Vol. 84 (1-2).

[43] Gilli, M., Grosse, S., Schumann, E. (2010) «Calibrating the NelsonSiegel Svensson model", COMISEF WORKING PAPERS SERIES, WPS-031 30/03/2010.

[44] Hladikova H., Radova J. (2012) «Term structure Modelling by Using Nelson Siegel Model", European Financial and Accounting Journal, Vol. 7, $\mathrm{n}^{\circ} 2$, Page 36-55.

[45] Hodges D. S., Parekh N., (2006) «Term Structure Slope Risk», The Journal of Fixed Income, Vol. 16 (3) 54-59

[46] Hubig (2012) «Introduction of a New Conceptual Framework for Government Debt Management - With a Special Emphasis on Modeling the Term Structure Dynamics ", Springer, 227 p.

[47] Hull J.C., White A. (1994) «Numerical Procedures for Implementing Term Structure Models II : Two-Factor Models", Journal of Derivatives, 37-48.

[48] Hyndman, R. J., Fan, Y. (1996) «Sample quantiles in statistical packages», American Statistician, Vol. 50, 361-365. 
[49] Jondeau E., Ricart R., (1998) "Le contenu en information de la pente des taux concernant l'évolution future des taux d'intérêt et de l'inflation en France", Bulletin de la Banque de France n ${ }^{\circ} 54$.

[50] Kovachev Y., Simeonov D., (2014) "Yield Curve Fitting with Data from Sovereign Bonds", ISBN 978-954-8579-53-7, Bulgarian National Bank.

[51] Lartey V.C., Li Y., (2018) «Zero-Coupon and Forward Yield Curves for Governement of Ghana Bonds", Sage Open, 1-15.

[52] Litterman, R., et J. Scheinkman (1991) "Common Factors Affecting Bond Returns", Journal of Fixed Income, vol. 1, n 1, p. 54-61.

[53] Marceau, E. (2013) «Modélisation et évaluation quantitative des risques en actuariat: Modèles sur une période», Springer.

[54] Martellini L., Priaulet P., Priaulet S., (2003) «Fixed-Income Securities: Valuation, Risk Management and Portfolio Strategies", Wiley.

[55] Martellini, L., Meyfredi, J.C., (2007) «A Copula Approach to Value-atRisk Estimation for Fixed-Income Portfolios", Journal of Fixed Income, Vol. 17, No 1, 5-15.

[56] McCulloch H. J., (1971) "Measuring the Term Structure of Interest Rates", The Journal of Business, Vol. 44, № 1, pp. 19-31

[57] McCulloch J.H, Kochin L.A., (1998) «The Inflation Premium Implicit in the US Real and Nominal Term Structures of Interest rates", Economics Department, Working Paper no 12, p. 98-12.

[58] McCulloch, J. H. (1975) "The Tax Adjusted Yield Curve», Journal of Finance, Vol 30, p. 811-29.

[59] McEnally, R.W. (1987) «The term structure of interest rate», The Handbook of Fixed Income Securities, p.1111-1150.

[60] McLeod, H.D. (1990) "The development of a market yield curve: the South Africa Solution", First AFIR International Colloquium, Paris, Vol. 2, p. 196-212.

[61] Molenaars T.K., Reinerink N.H., Hemminga M.A., (2015) «Forecasting the yield curve: art or science?», MPRA Paper No 63526.

[62] Muthoni L., Onyango S., Ongati O., (2015) «Extraction of Zero Coupon Yield Curve for Nairobi Securities Exchange: Finding the Best Parametric Model for East African Securities Markets ", Journal of Mathematics and Statistical Science, p. 51-74.

[63] Navas J. F. (2005) «Yield Curve Fitting with Term Structure Models: Empirical Evidence from the Euro Market ", Revista de Economia Aplicada, Vol. 13, n 39 . 
[64] Nelson C.R., Siegel A.F. (1987) «Parsimonious modelling of yield curves", Journal of Business, Vol. 60, 473-489.

[65] Paterson, A.A. (1996) «The JSE-Actuaries All Bond Indices - Two Flaws", Research paper, Alexander Paterson Faure Inc.

[66] R Development Core Team (2015) «R: A Language and Environment for Statistical Computing», Vienna, Austria, (R Foundation for Statistical Computing), ISBN : 3-900051-07-0.

[67] Roncalli T., (1998) «La structure par terme des taux zéro: Modélisation et implémentation numérique», Phd Thesis.

[68] Rosadi D., Nugraha A.Y., Dewi K.R., (2011) «Forecasting the Indonesian Government Securities Yield Curve Using Neural Networks and Vector Autoregressive model ", Bank for International Settlements.

[69] Rudebusch G. D., Wu T., (2008) "A macro-finance model of the term structure, monetary policy and the economy», The Economic Journal, Vol. 118 (530), p. 906-926.

[70] Seber, G.A.F., Wild, C.J. (2003) «Nonlinear Regression», Wiley Series in Probability and Statistics.

[71] Sedillot F., (1999) «La pente des taux contient-elle de l'information sur l'activité économique», Bulletin de la Banque de France nº 63.

[72] Shea, G.S. (1985) «Interest rate term structure estimation with exponential splines: A note», Journal of Finance, Vol. 40, p. 319-325.

[73] Smith, A., Wilson, T., (2001) «Fitting yield curves with long term constraints", Technical report, Bacon and Woodrow.

[74] Stambaugh, R.F. (1988) «The information in forward rates - Implications for models of the term structure", Journal of Financial Economics, Vol. 21, p. 41-70.

[75] Stander, S.Y. (2005) «Yield Curve Modeling», Palgrave Macmillian, 205 pages.

[76] Steeley, J.M. (1991) «Estimating the gilt-edged term structure: basis splines and confidence intervals", Journal of Business Finance and Accounting, Vol. 18, no 4, 513-529.

[77] Svensson. L.E.O (1994a) «Monetary policy with flexibles exchange rate and forward interest rates as indicators", Cahiers économiques et monétaires, Banque de France, Vol. 43, p. 305-332.

[78] Svensson. L.E.O (1994b) «Estimating and interpreting forward interest rates: Sweden 1992-1994", International monetary fund, IMF Working Paper, 1994/114. 
[79] Tanggaard, C. (1992) "Kernel smoothing of discount functions", Aarhus School of Business Working paper no 92-8.

[80] Tanggaard, C. (1997) "Nonparametric smoothing of Yield Curves", Review of Quantitative Finance and Accounting 9, 251-267.

[81] Vasicek, O., Fong, H. G. (1982) «Term structure modeling using exponential splines", The Journal of Finance, Vol. 37 (2), p. 339-348.

[82] Waggoner, D.F. (1997) «Spline Methods for Extracting Interest Rate Curves from Coupon Bond Prices", Federal Reserve Bank of Atlanta, Working Paper no 97-10.

[83] Wilkie D. (1986) «A Stochastic Investment Model for Actuarial Use», Transactions of the Faculty of Actuaries, 39:341-403.

[84] Wilkie D. (1995) "More on a Stochastic Model for Actuarial Use", British Actuarial Journal, p. 777-964.

[85] Zenios, S.A., Consiglio, A. (2016) «Risk Management Optimization for Sovereign Debt Restructuring", Journal of Globalization and Development, Vol. 6(2), p. 181-213

\section{NOTES}

1 L'auteur remercie les experts du cabinet FINAIR Conseil, dont les conseils ont permis d'améliorer la qualité de cet article.

Florent Gbongué, docteur en Sciences de gestion de l'université de Lyon, option: Actuariat. Par ailleurs, il est Enseignant-Chercheur en sciences économique et gestion. Contact: +225 47282072 / florent.gbongue@gmail.com

Université Félix-Houphouët-Boigny (UFHB) - 01 BP V34 Abidjan 01, Côte d'Ivoire.

FINAIR Conseil - 06 BP 6965 Abidjan 06. Contact : finairconseil@gmail.com

2 L'Union économique et monétaire ouest-africaine (UEMOA) est une organisation ouest-africaine créée le 10 janvier 1994 qui a comme mission la réalisation de l'intégration économique des États membres, à travers le renforcement de la compétitivité des activités économiques dans le cadre d'un marché ouvert et concurrentiel et d'un environnement juridique rationalisé et harmonisé. II comprend huit États membres: le Bénin, le Burkina Faso, la Côte d’Ivoire, la Guinée Bissau, le Mali, le Niger, le Sénégal et le Togo. http://www.uemoa.int/fr

3 La courbe des taux intéresse à la fois les économistes et les financiers. Les premiers s'intéressent aux anticipations futures des taux d'intérêt, qui influencent notamment les décisions monétaires, d'épargne et d'investissement. Par contre, les seconds l'utilisent pour valoriser et gérer les instruments financiers.

4 https://www.africanbondmarkets.org/fileadmin/uploads/afdb/pdf/Publications/Le_Guide_des_Courbes_ des_Taux_en_Afrique.pdf

5 Dans la suite de cet article, nous utiliserons le terme «courbe des taux» pour faire référence à la courbe des taux sans risque.

6 Selon les statistiques de l'Agence UMOA-Titres (2018), nous constatons que le volume annuel des émissions souveraines est passé de 2516 GXOF à 3330 GXOF (+32,4\%) sur la période 2014-2016, avant de reculer à 2644 GXOF (-20,6\%) en 2017. 
7 II n'existe pas une large base des investisseurs dans la zone UEMOA, composés en majorité par les banques locales et régionales ( $80 \%$ ). Aussi, le durcissement des conditions monétaires et l'application du dispositif bâlois ont réduit les ressources apportées par ces banques sur le marché des capitaux. Toutefois, la réduction des asymétries d'information pourrait élargir la base des investisseurs, notamment les investisseurs des pays occidentaux.

8 La CIMA est la Conférence interafricaine des marchés d'assurances. C'est un organisme de régulation du marché de l'assurance de 14 pays de l'Afrique subsaharienne qui sont Bénin, Burkina, Cameroun, Centrafrique, Comores, Congo (Brazzaville), Côte d'Ivoire, Gabon, Guinée Équatoriale, Mali, Niger, Sénégal, Tchad et Togo.

9 Dans Gbongué (2019), les facteurs de la courbe des taux sont mis en évidence. Dans le modèle de Nelson et Siegel, nous avons trois facteurs (niveau, pente et courbure). En outre, nous avons quatre facteurs dans le modèle de Svensson (niveau, pente, courbure 1 et courbure 2) et quatre facteurs dans le modèle de Björk et Christensen (niveau, pente 1, pente 2 et courbure).

10 En janvier 2016, le président Alassane Ouattara exhorte ses pairs à conduire la réflexion sur le développement du marché des capitaux et la réduction du coût de financement dans la zone UEMOA.

11 (cf. Bis [2005], Ecb [2008]).

12 En clair, l'auteur poursuit les travaux de Gbongué et Planchet (2015), en apportant quelques innovations méthodologiques. De manière pratique, il reformule le problème d'optimisation qui permet d'améliorer le calibrage des modèles candidats, tout en analysant la sensibilité des paramètres obtenus. De plus, il fait ressortir les facteurs de la courbe des taux sans risque, sans oublier de proposer une version dynamique de ces facteurs ainsi que les procédures de calibrage.

13 II s'agit des modèles de Nelson et Siegel et sa version élargie (Svensson), spline cubique, etc.

14 Cf. Bolder, D., Johnson G., Metzler A. (2004). An Empirical Analysis of the Canadian Term Structure of Zero-Coupon Interest Rates. Bank of Canada Working Paper No. 2004-48

15 Cf. Ricart R., Sicsic P., (1995). Estimation d'une structure par terme des taux d'intérêt sur données françaises. Bulletin de la Banque de France, n²22, Octobre 1995.

16 Dans le calcul de ce taux, nous prenons en compte trois dates: la date d'évaluation, et les dates de début et de fin du prêt ou de l'investissement.

$$
\begin{aligned}
17 R_{t}^{N S}(m)=\mathrm{L}_{t}+S_{t}\left[\frac{1-\exp \left(-\frac{m}{\tau_{1, t}}\right)}{\frac{m}{\tau_{1, t}}}\right]+\mathrm{C}_{t}\left(\left[\frac{1-\exp \left(-\frac{m}{\tau_{1, t}}\right)}{\frac{m}{\tau_{1, t}}}\right]-\exp \left(-\frac{m}{\tau_{1, t}}\right)\right. \\
18 R_{t}(m)=\mathrm{L}_{t}+S_{t}\left(\frac{1-\exp \left(-\frac{m}{\tau_{1, t}}\right)}{\left.\frac{m}{\tau_{1, t}}\right]+\mathrm{C}_{1, t}\left(\left[\frac{1-\exp \left(-\frac{m}{\tau_{1, t}}\right)}{\frac{m}{\tau_{1, t}}}\right]-\exp \left(-\frac{m}{\tau_{1, t}}\right)\right.}\right) \\
+\mathrm{C}_{2, t}\left(\left[\frac{1-\exp \left(-\frac{m}{\tau_{2, t}}\right)}{\left.\frac{m}{\tau_{2, t}}\right]-\exp \left(-\frac{m}{\tau_{2, t}}\right)}\right)\right.
\end{aligned}
$$


$\begin{aligned} & 19 R_{t}(m)=\mathrm{L}_{t}-S_{1, t}\left[\frac{1-\exp \left(-\frac{m}{\tau_{1, t}}\right)}{\frac{m}{\tau_{1, t}}}\right]+\mathrm{C}_{t}\left(\left[\frac{1-\exp \left(-\frac{m}{\tau_{1, t}}\right)}{\frac{m}{\tau_{1, t}}}\right]-\exp \left(-\frac{m}{\tau_{1, t}}\right)\right. \\ &-S_{2, t}\left[\frac{1-\exp \left(-\frac{2 m}{\tau_{1, t}}\right)}{\frac{2 m}{\tau_{1, t}}}\right]\end{aligned}$

20 Si ce modèle est proposé pour la zone UEMOA, en outre, il peut être appliqué dans plusieurs espaces économiques de l'Afrique.

21 Les facteurs de la courbe des taux (Level, Slope, Curvature) résument toute l'information contenue dans la courbe des taux sans risque. De plus, ils permettent d'appréhender la dynamique de cette courbe par la détermination de l'évolution temporelle de ces facteurs. Pour un exposé complet sur ce sujet, le lecteur pourra consulter les travaux de Diebold et Li (2006).

22 Ces coefficients doivent être strictement positifs. Pour les modèles de Nelson Siegel, Svensson et Bjork et Christensen, on a: $k_{1, t}=k_{2, t}=1$. De manière pratique, ils varient selon la date de cotation. Toutefois, ils doivent être fixés, afin de faciliter la prévision de la courbe, à l'image de Diebold et Li (2005).

23 Cf. Gbongué et Planchet (2015), Roncalli (1998), Martellini et al. (2003), Hladikova et Radova (2012).

24 II est perçu comme une mesure relative de variabilité ou de dispersion et est appelé risque unitaire en gestion quantitative des risques. Pour des informations complémentaires, l'auteur pourra consulter Marceau (2013).

25 C'est la date à laquelle l'obligation a été émise sur le marché financier.

26 II s'agit de la date de paiement des coupons.

27 C'est la date de remboursement du capital par l'émetteur.

28 C'est la somme du prix coté et de l'intérêt couru.

29 C'est la différence entre les dates de maturité et de cotation.

30 C'est la différence entre les dates de maturité et d'émission.

31 La construction de la courbe des taux sans risque de référence de l'UEMOA est justifiable dans une union économique et monétaire.

32 Cette valeur est déterminée sur des hypothèses cohérentes de croissance et d'inflation sur le long terme. Sur recommandation des experts (directeurs des activités de marché, etc.), nous avons choisi un taux de croissance réel de 4,2\% et un taux d'inflation de $2 \%$. En outre, ces choix sont corrigés progressivement de sorte que la courbe de taux finale obtenue permette in fine de retrouver les données d'un émetteur de référence en l'occurrence la Côte d'Ivoire, car il est le moteur de la croissance économique de la zone UEMOA. Cette approche est retenue dans la pratique pour la construction des courbes des taux. Par exemple, les hypothèses retenues dans la zone euro sont: taux de croissance réel long terme $(2,2 \%)$ et inflation (2\%).

33 Voir http://www.bceao.int/Instruments-de-mise-en-oeuvre-de.html

34 Sur la période 2008-2017, le taux de croissance réel moyen dans la zone UEMOA est de 5,24\%, tandis que l'inflation moyenne s'établit à 1,27\%.

35 La relation suivante doit être vérifiée : $\left(1+R_{t}(m)\right)^{m}=\left(1+R_{t}(1)\right) \prod_{k=1}^{m-1}\left(1+F_{t}(k, 1)\right)$.

36 Cette situation s'explique par le fait que les taux à terme sont en réalité les anticipations futures des taux sans risque, auxquels nous ajoutons les primes à terme.

37 La prise en compte des facteurs de la courbe des taux dans l'analyse des politiques peut être consultée dans Rudebusch G. D., Wu T., (2008); Wu T., (2002); Diebold F.X., Rudebusch G.D., Aruoba B., (2006); etc. 
38 Le test de Jarque Bera rejette l'hypothèse de normalité des erreurs d'estimation des prix des obligations souveraines.

39 Sur la période 2012-2017, les écarts types sont de 28,13\% et 45,71\% respectivement pour la Theil-U-Statistic et la MAPE.

40 L'émetteur de l'obligation paie plus cher lorsque la maturité est lointaine.

41 II s'agit de la différence entre le taux à 10 ans et le taux à 1 an.

42 Cette baisse peut s'expliquer par une hausse des taux court terme dans la zone UMOA, qui est une réponse naturelle au durcissement des conditions monétaires.

43 Le développement du marché des capitaux et les politiques monétaire et budgétaire. 


\section{ANNEXES}

FIGURE 5 Histogramme des variations des rendements obligataires à 1, 2, 3, 5, 7 et 10 ans.

Histogramme des variations des rendements obligataires à 1 ans sur la période 2012-2017

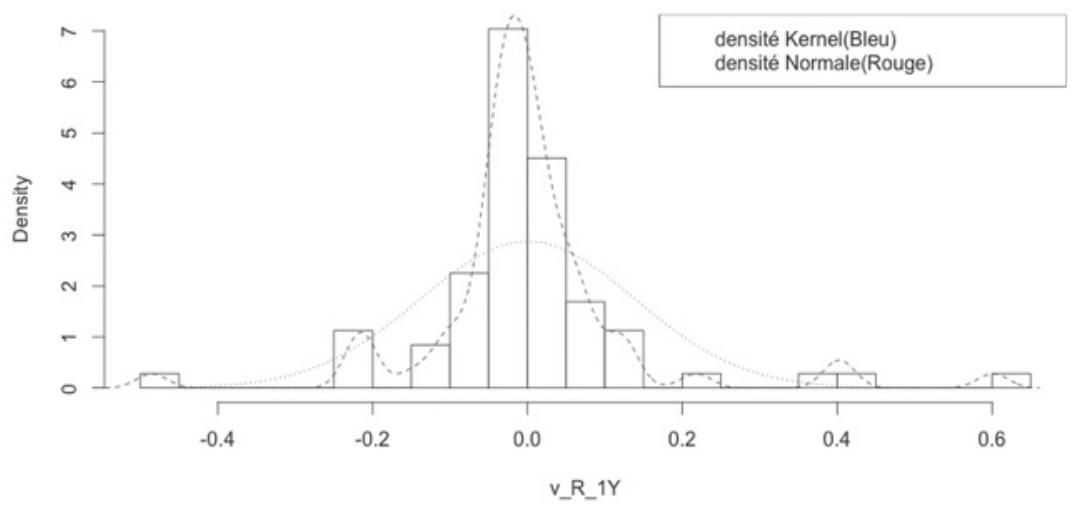

Histogramme des variations des rendements obligataires à 2 ans sur la période 2012-2017

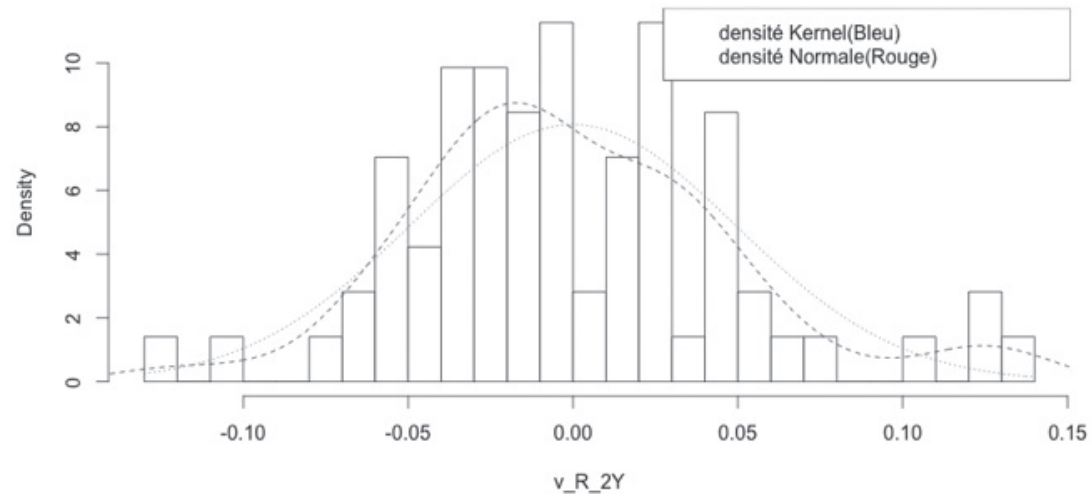

Histogramme des variations des rendements obligataires à 3 ans sur la période 2012-2017

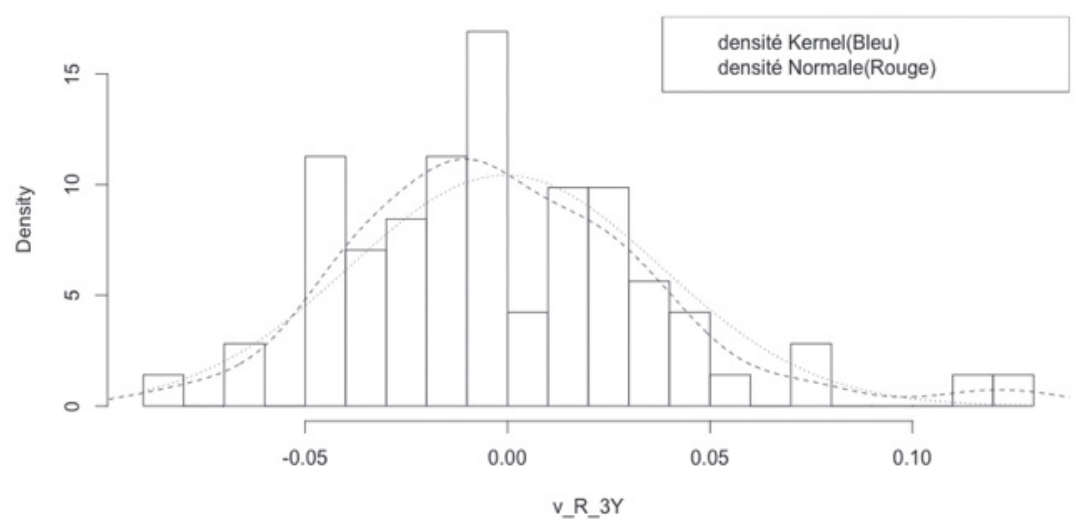




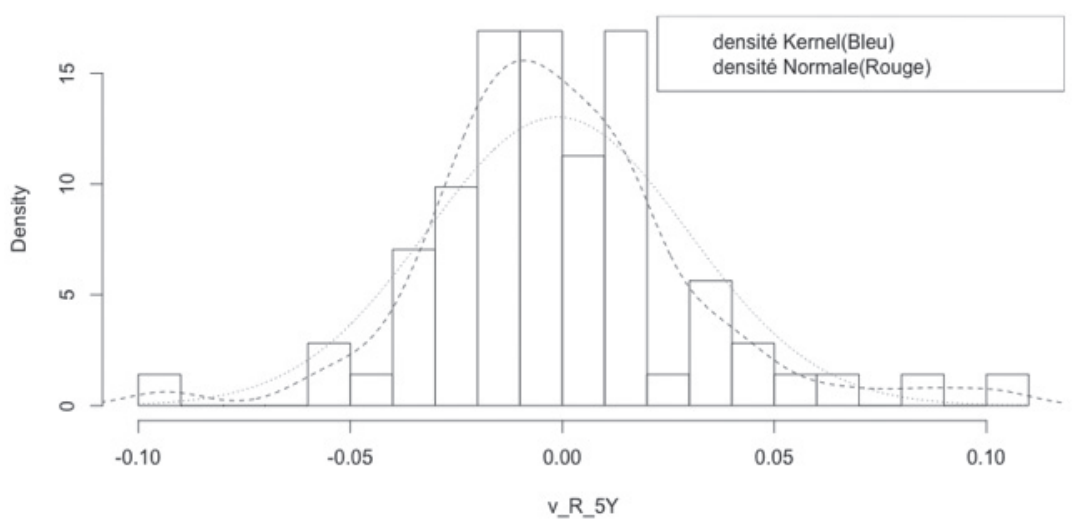

Histogramme des variations des rendements obligataires à 7 ans sur la période 2012-2017

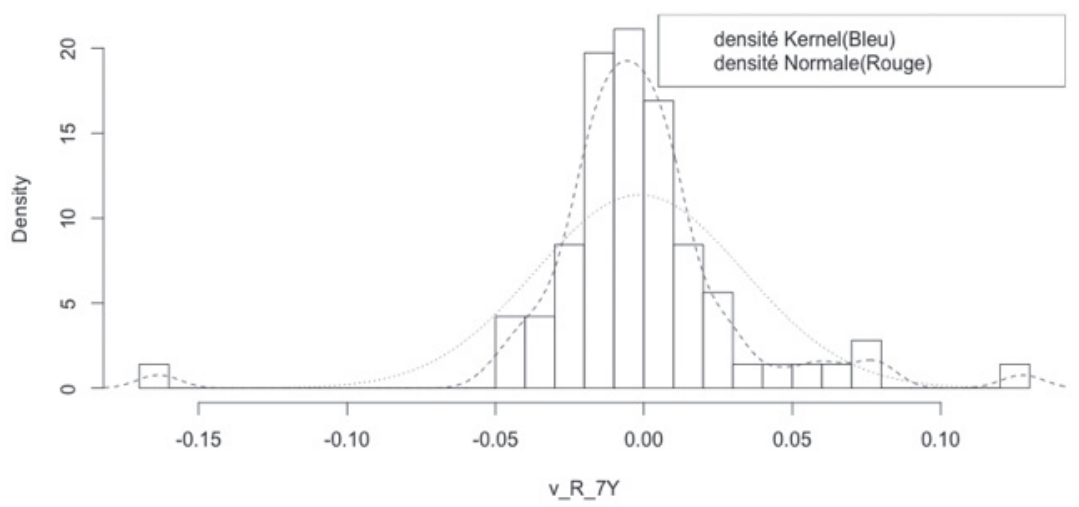

Histogramme des variations des rendements obligataires à 10 ans sur la période 2012-2017

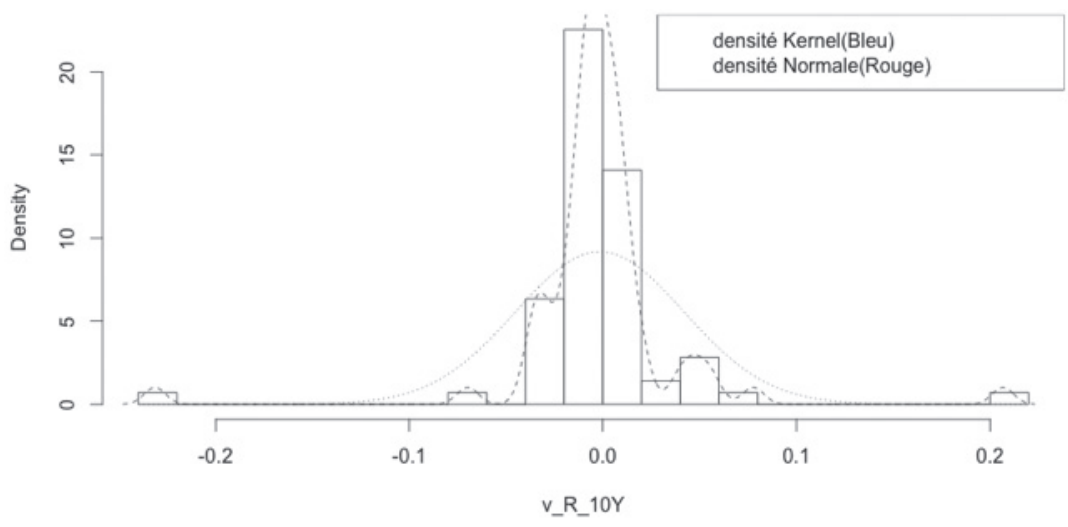


FIGURE 6 Évolution des trois facteurs de la courbe de rendement zéro-coupon, provenant du nouveau modèle dans la zone UEMOA

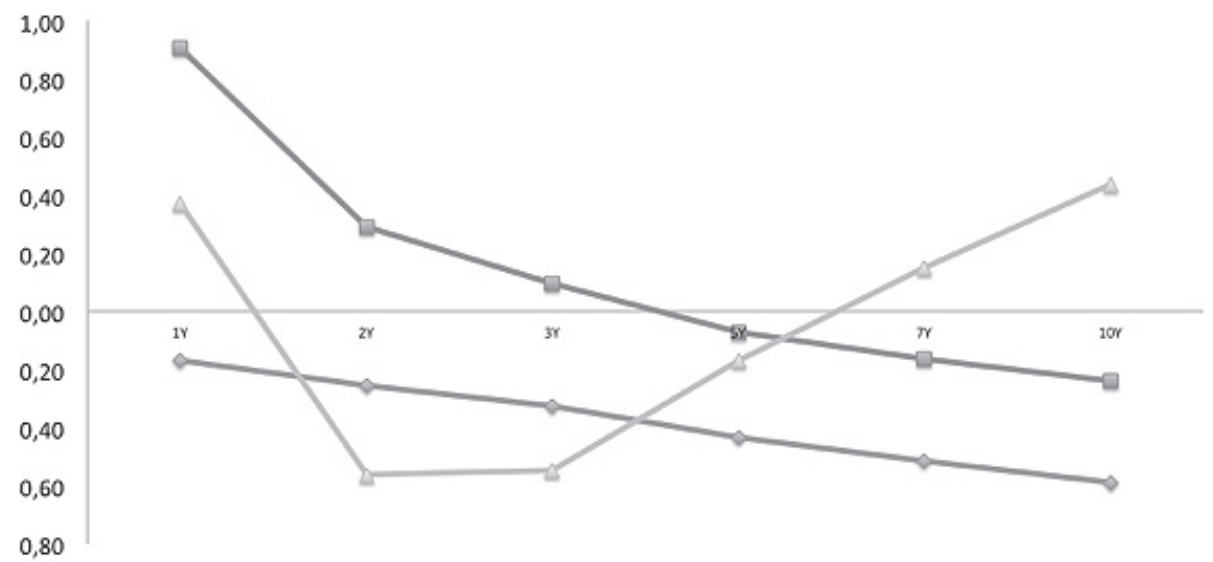

$\approx-\mathrm{PC} 1 \_51,3 \% \quad \square-\mathrm{PC} 2 \_36,7 \% \quad-\mathrm{PC} 3 \_11,7 \%$ 
FIGURE 7 Histogramme des erreurs d'estimation des prix des obligations souveraines sur la période 2012-2017

Histogramme des erreurs d'estimation des prix (U.statistic) sur la période 2012-2017

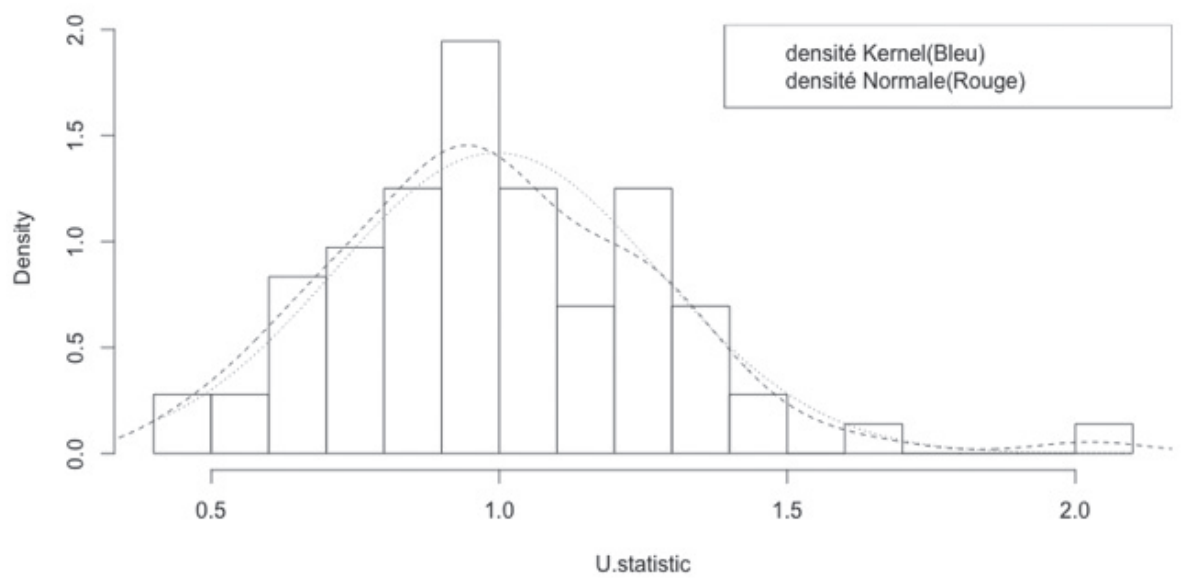

Histogramme des erreurs d'estimation des prix (MAPE) sur la période 2012-2017

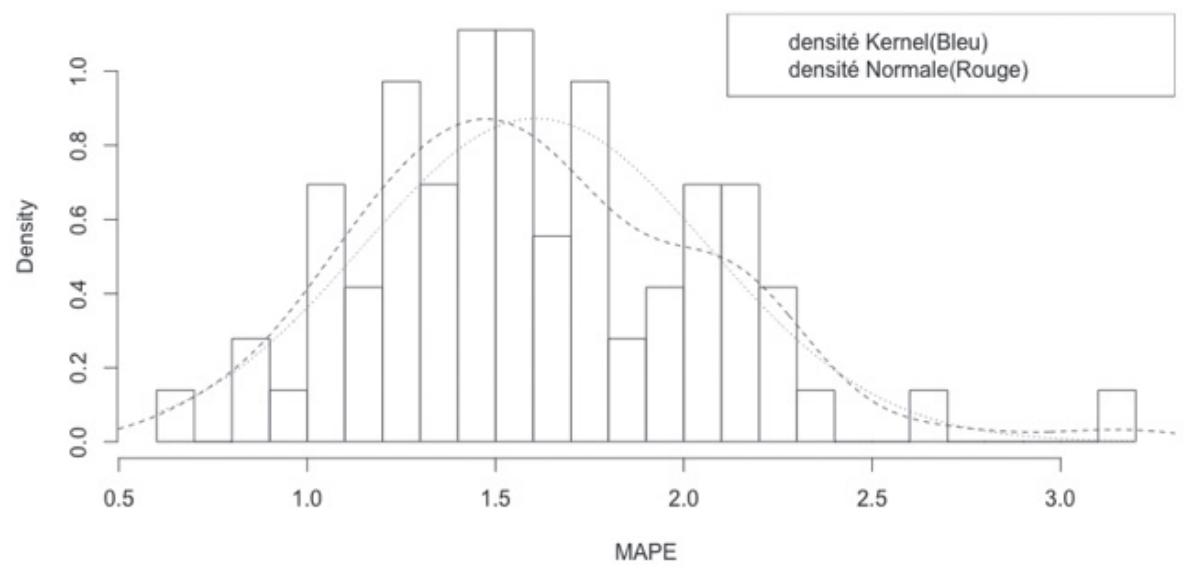


FIGURE 8 Évolution des erreurs d'estimation mensuelles des prix (U, MAPE) sur la période 2012-2017

Evolution des erreurs d'estimation des prix sur la période 2012-2017

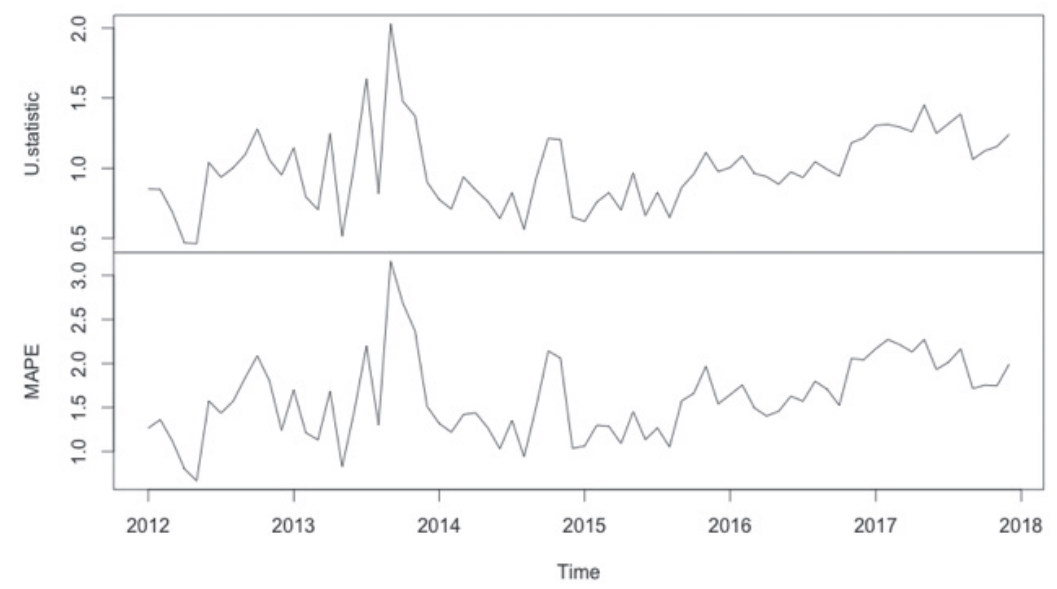

